\title{
Alfvén ion cyclotron waves in sheath regions driven by interplanetary coronal mass ejections
}

\author{
Matti Ala-Lahti ${ }^{1}$, Emilia K. J. Kilpua ${ }^{1}$, Jan Souček ${ }^{2}$, Tuija I. Pulkkinen ${ }^{3,4}$, and \\ Andrew P. Dimmock ${ }^{5}$ \\ ${ }^{1}$ Department of Physics, P.O. Box 64, University of Helsinki, Helsinki, Finland \\ ${ }^{2}$ Institute of Atmospheric Physics, Czech Academy of Sciences, Prague, Czech Republic \\ ${ }^{3}$ Department of Climate and Space Sciences and Engineering, University of Michigan, Ann Arbor, MI, USA \\ ${ }^{4}$ Aalto University, School of Electrical Engineering, Espoo, Finland \\ ${ }^{5}$ Swedish Institute of Space Physics, Uppsala, Sweden
}

\section{Key Points:}

- AIC waves are frequent structures in sheath regions driven by ICMEs.

- AIC waves tend to have their frequency below the ion cyclotron frequency.

- AIC waves are observed in lower ion $\beta_{\|}$plasma than mirror mode waves.

This is the author manuscript accepted for publication and has undergone full peer review but has not been through the copyediting, typesetting, pagination and proofreading process, which may lead to differences between this version and the Version of Record. Please cite this article as doi: 10.1029/2019JA026579

Corresponding author: Matti Ala-Lahti, matti.ala-lahti@helsinki .fi

This article is protected by copyright. All rights reserved. 


\begin{abstract}
We report on a statistical analysis of the occurrence and properties of Alfvén ion cyclotron [AIC] waves in sheath regions driven by interplanetary coronal mass ejections [ICMEs]. We have developed an automated algorithm to identify AIC wave events from magnetic field data and apply it to investigate 91 ICME sheath regions recorded by the Wind spacecraft. Our analysis focuses on waves generated by the ion cyclotron instability. AIC waves are observed to be frequent structures in ICME-driven sheaths, and their occurrence is the highest in the vicinity of the shock. Together with previous studies, our results imply that the shock compression has a crucial role in generating wave activity in ICME sheaths. AIC waves tend to have their frequency below the ion cyclotron frequency, and, in general, occur in plasma that is stable with respect to the ion cyclotron instability and has lower ion $\beta_{\|}$than mirror modes. The results suggest that the ion beta anisotropy $\beta_{\perp} / \beta_{\|}>1$ appearing in ICME-sheaths is regulated by both ion cyclotron and mirror instabilities.
\end{abstract}

\title{
1 Introduction
}

Interplanetary coronal mass ejections [ICME; e.g., Kilpua et al., 2017a] are interplanetary counterparts of CMEs [e.g., Webb and Howard, 2012; Chen, 2017], the most drastic eruptions of the Sun, ploughing through the ambient solar wind often so fast that they exceed the local magnetosonic speed. As a consequence, a shock and sheath form upstream of the ICME itself. At the Earth, all these individual plasma structures are known to drive geomagnetic activity [e.g., Tsurutani et al., 1988; Zhang et al., 2007; Kilpua et al., 2017b].

Since their discovery in 1970s, a number of observational and simulation studies have aimed at deepening our understanding on CMEs and ICMEs. Especially, research has focused on the ones with a flux rope configuration [e.g., Burlaga et al., 1981; Burlaga, 1988; Bothmer and Schwenn, 1998; Möstl et al., 2012; Isavnin et al., 2014; Nieves-Chinchilla et al., 2016, 2018; Démoulin et al., 2018; Good et al., 2018; Palmerio et al., 2018]. However, a considerable fraction of ICME-driven space weather storms are purely induced by sheaths or have a significant sheath-contribution. ICME sheaths are particularly capable of driving strong geomagnetic activity in the high-latitude magnetosphere of the Earth [e.g., Huttunen et al., 2002; Lugaz et al., 2016; Kilpua et al., 2017b]. In addition, the compression processes happening in the ICME sheath can have an effect on the geoefficiency of the following ICME by enhancing the magnetic reconnection occurring at the leading edge of the ICME [Feng and Wang, 2013; Ruffenach et al., 2015]. ICME-driven sheaths have highly turbulent internal structure, and heating of plasma may occur within the sheaths for example due to the shock [e.g., Kataoka et al., 2015; Guo et al., 2017] and field line draping [e.g., Gosling and McComas, 1987; Kaymaz and Siscoe, 2006; Siscoe et al., 2007].

The heating mechanisms in action at the shock produce ion beta anisotropy $\beta_{\perp} / \beta_{\|}>1$, where $\beta$ expresses the ratio of thermal pressure to magnetic pressure and subscripts $\perp$ and \| denote components perpendicular and parallel to the background magnetic field, in plasma. In the Earth's magnetosheath, the enhanced anisotropy is known to drive mirror [Hasegawa, 1969] and ion cyclotron [IC; Weibel, 1970; Davidson and Ogden, 1975] instabilities that regulate plasma by generating intense magnetic field fluctuations and keeping the plasma at the state of marginal stability [e.g., Kaufmann et al., 1970; Crooker and Siscoe, 1977; Fuselier et al., 1994; Hellinger et al., 2003, 2006; Remya et al., 2013; Souček et al., 2015]. Both instabilities tend to grow under similar plasma conditions and for bi-Maxwellian ions and cold electrons, plasma is considered mirror unstable when the condition $\frac{\beta_{\perp}}{\beta_{\|}}>1+\frac{1}{\beta_{\perp}}$ holds [Hasegawa, 1969; Davidson and Ogden, 1975; Hellinger, 2007]. These instabilities compete with each other, and according to linear approximation, the IC instability should dominate in lower beta plasma [e.g., Gary, 1992; Gary et al., 1993; Shoji et al., 2009, 2012; Remya et al., 2013].

Waves generated by these two instabilities are mirror mode [MM] waves and Alfvén ion cyclotron [AIC] waves, which are also often called electromagnetic ion cyclotron [EMIC] waves, and their occurrence indicates that the related instabilities have taken place to dissipate the excess anisotropy [e.g., Song et al., 1994; Bale et al., 2009; Gary et al., 2016]. While AICs and MMs have been studied 
widely in the solar wind [e.g., Zhang et al., 2008, 2009; Jian et al., 2009, 2010, 2014; Wicks et al., 2016; Zhao et al., 2017, 2019] and in the Earth's magnetosheath [e.g., Anderson and Fuselier, 1993; Anderson et al., 1994; Souček et al., 2008; Génot et al., 2009; Dimmock et al., 2015; Osmane et al., 2015] there are only a few studies on wave properties of the ICMEs and their sheath regions [e.g., Liu et al., 2006; Kajdič et al., 2012; Siu-Tapia et al., 2015; Blanco-Cano et al., 2016; Ala-Lahti et al., 2018].

ICME-driven sheaths are exceptional plasma environments due to the small deflection speed of the flow in the sheath. As a consequence, plasma tends to pile in front of the ICME maintaining the record of previous interactions [e.g., Siscoe and Odstrcil, 2008]. At 1 AU, mirror unstable plasma has been reported in sheaths driven by ICMEs [Liu et al., 2006], and MM waves are observed throughout the ICME sheath [Ala-Lahti et al., 2018]. However, Ala-Lahti et al. [2018] showed that although the plasma in ICME sheaths has enhanced anisotropy $\beta_{\perp} / \beta_{\|}$, it is generally only marginally mirror unstable and the majority of observed MMs occur in mirror stable plasma at $1 \mathrm{AU}$ [Ala-Lahti et al., 2018]. The authors suggested that MMs [almost exclusively dip-like] in mirror stable plasma could have formed at earlier times of the ICME sheath evolution when the plasma was in a mirror unstable state.

In the Earth's magnetosheath, the highest occurrence of AIC waves is reported in the downstream of a weak [Alfvén Mach number $M_{A}<6$ ] quasi-perpendicular [shock angle $\theta_{B n}>45^{\circ}$ ] bow shock [Souček et al., 2015]. As such shocks often precede ICME-driven sheaths, and since the sheath plasma typically shows higher values of anisotropy $\beta_{\perp} / \beta_{\|}$, the conditions should be favorable for the growth of AIC waves as suggested by Ala-Lahti et al. [2018].

To improve our understanding of ICME sheath regions and the competition between mirror and IC instabilities, in this article we investigate the occurrence of AIC waves within ICME sheaths. We investigate what plasma conditions are associated with AIC waves and compare our results with the previously published results on mirror modes in ICME sheaths [Ala-Lahti et al., 2018]. The article is organized by introducing the used data sets and developed methods in Section 2, presenting the statistical results of AIC wave occurrence in Section 3, and finally discussing the results and drawing conclusions in Section 4.

\section{Data and Methods}

\subsection{Data Sets}

In our statistical analysis, we use the same data set as in Ala-Lahti et al. [2018] and originally generated by Palmerio et al. [2016]. In total, 91 ICME-driven sheath regions are investigated, and similarly to Palmerio et al. [2016], we divide the ICME sheaths into three sub-regions according to a fractional distance parameter $[F]$ that gives the relative location in the sheath with values between zero [shock] and one [leading edge of the ICME ejecta]. The sub-regions are termed near-shock $[0<F<1 / 3]$, mid-sheath $[1 / 3<F<2 / 3]$ and near leading edge [near-LE; $2 / 3<F<1]$ regions.

We investigate the occurrence of AIC waves in the ICME sheaths by using measurements of the Wind spacecraft, positioned close to the L1 Lagrangian point after the launch in November 1994, with an exception of a complex trajectory between 1999 and 2004 traversing in a petal-shaped trajectory through the Earth's magnetosphere and solar wind. Here, we use only periods when the spacecraft was in the solar wind. We use high resolution magnetic field data from the Wind Magnetic Fields Investigation [MFI] instrument [Lepping et al., 1995] and the proton velocity vector $\left[\overrightarrow{v_{p}}\right]$, number density $\left[n_{p}\right]$ and thermal speed data, both parallel $\left[v_{\|}\right]$and perpendicular $\left[v_{\perp}\right]$ to the magnetic field, from the Wind Solar Wind Experiment [SWE] instrument [Ogilvie et al., 1995]. The data are obtained from the NASA Goddard Space Flight Center Coordinated Data Analysis Web [CDAWeb, http://cdaweb.gsfc.nasa.gov/].

Although the time resolution of the magnetic field data depends on the mode of the MFI instrument and varies between $0.046 \mathrm{~s}$ and $1.84 \mathrm{~s}, 97.5 \%$ of the data in this study have the time resolution of $0.092 \mathrm{~s}$. To analyze the Doppler effect and study the plasma conditions around AIC 
waves, we use SWE data registered approximately every $90 \mathrm{~s}$ and compute $5 \mathrm{~min}$ averages of ion $\beta_{\|}$ and $\beta_{\perp}, \overrightarrow{v_{p}}$ and $v_{\perp}$ [see Ala-Lahti et al., 2018].

For the approximate ion cyclotron instability threshold, we use the relation [Hellinger et al., 2006]

$$
\frac{\beta_{\perp}}{\beta_{\|}}=1+\frac{0.43}{\left(\beta_{\|}+0.0004\right)^{0.42}} .
$$

Furthermore, the results of the AIC wave event identification procedure discussed below are compared to the observed mirror modes in ICME-driven sheath regions reported by Ala-Lahti et al. [2018], where the identification algorithm of mirror modes is described in detail.

\subsection{Alfvén Ion Cyclotron [AIC] Wave Event Identification}

In this study, we have constructed an automated algorithm to identify AIC waves from spaceborne magnetic field measurements. The identification is based on a transverse left-hand polarization and quasi-parallel propagation direction $[\vec{k}]$ with respect to the background magnetic field $[\vec{B}]$, i.e., $\theta_{k B}<45^{\circ}$. These are characteristic properties of AIC waves in the Earth's magnetosheath [Remya et al., 2014]. In addition, we have applied a technique called Rosetta automatic wave analysis [RAWA; Tsurutani et al., 2013; Remya et al., 2014, 2015] and methods used by Souček et al. [2015].

First, we derive wave fields $\delta \vec{B}$ from the background magnetic field by performing high-pass filtering with standard fast Fourier transform on the data at $15 \mathrm{mHz}$ that is derived empirically for the Wind spacecraft data and previously used by Remya et al. [2014]. The remaining low-pass data give the background magnetic field $[\vec{B}]$ with respect to which we compute parallel and perpendicular components of the wave field, i.e., fluctuations as $\delta \vec{B}_{\|}=\left(\delta \vec{B} \cdot \hat{b_{0}}\right) \hat{b_{0}}$ and $\delta \vec{B}_{\perp}=\delta \vec{B}-\hat{b_{0}} \delta B_{\|}$, where $\hat{b_{0}}$ is the unit vector of $\vec{B}$.

To identify the AIC wave activity from the fluctuations, we investigate every non-overlapping 1 min data interval having a maximum time gap between two successive data points of $1.84 \mathrm{~s}$ and a maximum cumulative time gap within the interval of $3.68 \mathrm{~s}$. For every 1 min interval passing these criteria, we compute the mean square fluctuations

$$
\begin{gathered}
\delta B_{\|}^{2}=\frac{1}{N} \sum_{i=1}^{N} \delta B_{\|, i}^{2} \\
\delta B_{\perp}^{2}=\frac{1}{N} \sum_{i=1}^{N} \delta B_{\perp, i}^{2},
\end{gathered}
$$

where $N$ is the number of data points within an interval. In addition, we compute the ratios $\frac{\delta B_{\|}^{2}}{\bar{B}^{2}}$ and $\frac{\delta B_{\perp}^{2}}{\bar{B}^{2}}$, where $\bar{B}^{2}=\frac{1}{N} \sum_{i=1}^{N} B_{i}^{2}$ is the mean square background magnetic field, and perform the minimum variance analysis [MVA; Sonnerup and Cahill, 1967].

The MVA has been widely used in statistical studies of different wave modes in different sheath regions in the Solar System [see e.g., Tsurutani et al., 2013; Remya et al., 2014, 2015; Dimmock et al., 2015; Osmane et al., 2015; Souček et al., 2015; Ala-Lahti et al., 2018, and references therein]. The method yields the maximum $\left[\vec{B}_{1}\right]$, intermediate $\left[\vec{B}_{2}\right]$ and minimum $\left[\vec{B}_{3}\right]$ variance directions with corresponding eigenvalues $\left[\lambda_{1}, \lambda_{2}\right.$ and $\left.\lambda_{3}\right]$ representing the actual variances. The variance directions can be used to form a right-handed coordinate system, where $\vec{B}_{1} \times \vec{B}_{2}=\vec{B}_{3}$. We consider the minimum variance direction as the propagation direction of waves $\left[\vec{k} \| \vec{B}_{3}\right.$; Verkhoglyadova et al., 2010; Remya et al., 2014].

The identification algorithm omits intervals during which the azimuthal and polar directions of $\vec{B}$ change more than $30^{\circ}$, and also highly elliptically and linearly polarized events are omitted by requiring $\lambda_{1} / \lambda_{2}<10$ [CR1]. Similarly to Souček et al. [2015], we identify a 1 min interval 
as a possible AIC wave event [AIC event; AIC] if the angle $\left[\theta_{k B}\right]$ between the wave vector $\vec{k}$ and the background magnetic field $\vec{B}$ is quasi-parallel, and the perpendicular fluctuation amplitude is sufficiently large compared to the background magnetic field and the parallel fluctuations within the interval. These criteria are listed as

Criterion CR2 is based on the results and methods of previous studies [Remya et al., 2014; Souček et al., 2015], whereas CR3 and CR4 are modified from the identification procedure of AIC wave events given by Souček et al. [2015]. We have decreased the threshold of CR3 by one order of magnitude from the one used by Souček et al. [2015], because of the high-pass filtering performed at the beginning of our analysis. Souček et al. [2015] obtained fluctuations directly from the magnetic field data and the spectrum of fluctuations was not limited, whereas we investigate the occurrence of AIC events only above the frequency threshold of $15 \mathrm{mHz}$. The threshold of CR4, is on the other hand, increased in our study from the value used by Souček et al. [2015], who required $\frac{\delta B_{\perp}^{2}}{\delta B_{\perp}^{2}}>2$. The value of 8.6 is based on our investigation of the interval initially studied by Remya et al. [2014] to which we refer to from now on as the 'Remya interval'.

The Remya interval was measured by the Wind spacecraft in the Earth's magnetosheath at 01:52-02:26 UT on 18 August 1999, and AIC waves are identified throughout the event [Remya et al., 2014]. We investigated every non-overlapping 1 min interval within the Remya interval and compute the distribution of the ratio $\frac{\delta B_{\perp}^{2}}{\delta B_{\|}^{2}}$. The lower quartile of the distribution is 8.6, which we select as the threshold value of our AIC wave event identification procedure. Filtering was also performed by Remya et al. [2014], and as AIC waves are transverse, perpendicular fluctuations can be expected to dominate parallel fluctuations similarly also in ICME-driven sheaths in the highfrequency domain $[>15 \mathrm{mHz}]$. On the other hand, the magnitude of the background magnetic field might vary strongly within an ICME sheath [e.g., Kilpua et al., 2017a] and differs from the one of the Earth's magnetosheath, which is why we prefer modifying the threshold used by Souček et al. [2015] over applying statistical values of the Remya interval.

Figure 1 illustrates how the criteria limit the magnetic field data of ICME-driven sheath regions in our data set. The panels show that whereas majority of the data pass CR1 and CR2 whereas the criteria concerning the magnitude of perpendicular fluctuations of the magnetic field omits most of the data.

In the end, our identification procedure of AIC events considers the origin of wave events. In our procedure, we omit events where the observed waves are likely to be generated by the parallel fire hose [FH] instability [Quest and Shapiro, 1996; Gary et al., 1998], which also generates transverse waves propagating quasi-parallel with respect to the background magnetic field [e.g., Jian et al., 2014; Zhao et al., 2019, and references therein]. In this study, the FH instability threshold is computed similarly as in Eq. 1 but by using the coefficients for the parallel fire hose instability given by Hellinger et al. [2006]. Finally, we classify AIC events as events that are stable with respect to the FH instability being shifted $33 \%$ into the direction of FH stable plasma. The shifted FH instability threshold curve is illustrated with the light purple curve in Fig. 2, which shows the relative frequency of all plasma observations in ICME-driven sheath regions in two-dimensional space of $\beta_{\perp} / \beta_{\|}$and $\beta_{\|}$. Events being located below the light purple curve are omitted from analysis. In addition, thresholds of IC [black], mirror [yellow] and FH [dark purple] instabilities are overplotted in Fig. 2. The figure illustrates that $26 \%$ of data points are below the shifted FH instability curve, whereas $11 \%$ are unstable with respect to the IC instability, and plasma is only marginally mirror unstable. 
Similarly to Jian et al. [2009, 2010], the polarization of the identified AIC events is examined by analyzing the quadrature spectrum [Fowler et al., 1967; Rankin and Kurtz, 1970; Means, 1972]. We compute the cross power spectral density in the right-handed coordinate system of the variance directions, and the resulting spectral matrix is used to compute the polarization of the wave event, with a positive [negative] sign denoting right [left]-handed [RH and LH, respectively] polarization. We tested the validity of this method successfully on the waves observed within the Remya interval before analyzing AIC events in ICME-driven sheath regions.

We further compute the angular frequency of AIC events in the spacecraft frame $\left[\omega_{s c}\right]$ by using the frequency averaged with a weight of the power. To investigate the underlaying nature of AIC events in the solar wind frame, we have to estimate possible Doppler effects. We obtain the angular frequency of an AIC event in the solar wind frame $\left[\omega_{s w}\right]$ from the dispersion relation [Davidson and Ogden, 1975]

$$
-(k c)^{2}-\omega_{p e}^{2} \frac{\omega_{s w}}{\Omega_{e}}-\omega_{p i}^{2} \frac{\omega_{s w}}{\omega_{s w}+\Omega_{i}}\left[1+\frac{1}{2} \frac{\left(k\left|\vec{v}_{\perp}\right|\right)^{2}}{\omega_{s w}\left(\omega_{s w}+\Omega_{i}\right)}\right]=0,
$$

where $k$ is the wave number, $c$ is the speed of light, $\omega_{p e}$ and $\omega_{p i}$ are the electron and ion plasma frequencies, $\Omega_{e}$ and $\Omega_{i}$ the electron and ion cyclotron frequencies, and $\left|\vec{v}_{\perp}\right|$ is the thermal velocity of the proton perpendicular to the background magnetic field, by substituting $k=\omega_{s w} / V_{p h}$, where $V_{p h}$ is the theoretical phase speed of a wave event, and using Doppler-shift relation [Tsurutani et al., 1983]

$$
\omega_{s w}=\omega_{s c}\left(1+\frac{V_{s w}}{V_{p h}} \cos \theta_{k V_{s w}}\right)^{-1},
$$

where $V_{s w}$ is the solar wind velocity $\left[V_{s w}=\left|\overrightarrow{v_{p}}\right|\right]$ and $\theta_{k V_{s w}}$ is the angle between $\vec{k}$ and $\overrightarrow{v_{p}}$, to express $V_{p h}$. After solving $\omega_{s w}$, we use Eq. 5 to compute $V_{p h}$.

As noted by Remya et al. [2014], the MVA has $180^{\circ}$ uncertainty in the absolute direction of $\vec{k}$ [Sonnerup and Cahill, 1967; Tsurutani et al., 1983]. Because the electric field measurements required for the determination of the absolute direction are unavailable, we assume that AIC events are propagating in the direction of the solar wind flow in Eq. 5 and study the ratio $V_{s w} / V_{p h}$. AIC events propagating in the solar wind flow direction maintain their sense of rotation and are Dopplershifted to higher frequencies. $V_{s w} / V_{p h}>1$ implies that instead of being LH polarized in the solar wind frame, AIC events propagating against the flow might be observed having RH polarization in the spacecraft frame [Remya et al., 2014].

We show two examples of AIC events identified by the algorithm in Fig. 3, and their variables and the frequencies normalized to the ion cyclotron frequency in Table 1. Both of the events are RH polarized in the spacecraft frame, and the event in Fig. 3a has $\omega_{s c} / \Omega_{i}>1$. However, in the solar wind frame, both have frequencies below the ion cyclotron frequency and could be $\mathrm{LH}$ polarized due to $V_{s w} / V_{p h}>1$.

\section{Statistical Results}

\subsection{Occurrence of AIC Events}

We now use the methods described in Section 2 to study AIC occurrence in sheath regions driven by ICMEs. In total, our study includes 3303 one-minute intervals that are classified as AIC events. They correspond $6 \%$ of all examined intervals, and the corresponding occurrence rate within individual sheaths shown in Fig. $4 \mathrm{a}$ varies between $1 \%$ and $29 \%$ with the median of 5\%.

At least two AIC events are observed in every studied sheath region, and the maximum number of AICs identified within one sheath is 180 . Figure $4 \mathrm{~b}$ shows the distribution of the number of ICME-driven sheath regions as a function of the number of AICs identified within a sheath and the median, lower and upper quartiles [LQ and UQ] of the distribution. The distribution is weighted around the LQ [18 AICs] and median [27 AICs]. Above the UQ [43 AICs] , the size of a bin varies between zero and two ICME sheaths with the exception of three bins. We note that $45 \%$ of all AICs 


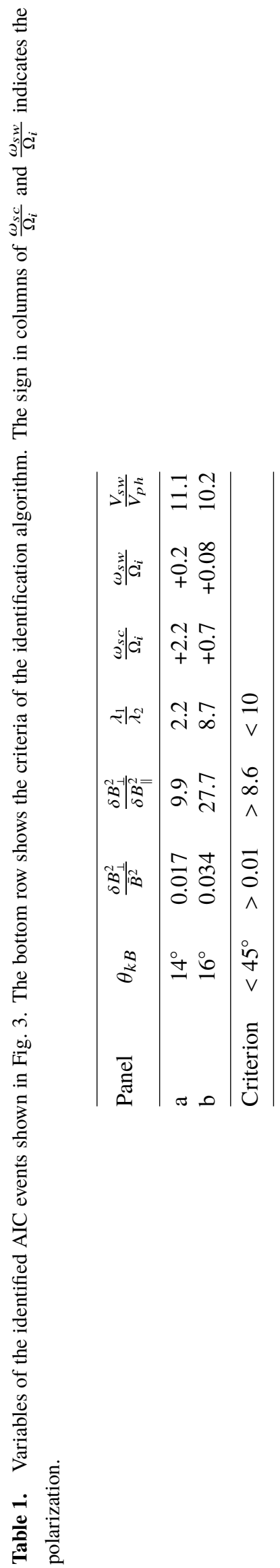

This article is protected by copyright. All rights reserved. 
are observed in the sheaths that have less than 43 AICs. The numerical values of the quartiles shown in Fig. $4 \mathrm{~b}$ and in addition, the percentage of the sheaths having more AICs than the median are given in the top row of Table 2.

In the upper part Table 2, the quartiles of the number of AICs in different sub-regions of ICMEdriven sheaths and the percentage of how many sub-regions in question have more than 27 AICs are given. Near-shock region has the highest value of each quartile and also the highest percentage of having $>27$ AICs. In addition, the values decrease monotonically towards the end of the ICME sheath. We note that $50 \%$ of all AIC events occur in the near-shock region whereas $26 \%$ and $24 \%$ located in the mid-sheath and near-LE regions.

We examine the statistical significance of the difference in the total number of AICs between the sub-regions in the lower part of Table 2. The average number of AICs in an ICME sheath is $36 \pm 3$ events, and like the quartiles, also the average of a sub-region decreases from near-shock towards the leading edge of an ICME. Furthermore, p-values indicating statistical significance [Welch, 1938] imply that the difference in the averages between the near-shock region and other sub-regions is statistically significant. The p-values $1.5 \cdot 10^{-4}$ and $2.5 \cdot 10^{-5}$ of the near-shock - mid-sheath regions and the near-shock - near-LE regions are noticeably below the nominal significance level 0.05 [see for example Ruxton, 2006]. The difference between the mid-sheath and near-LE regions is, however, insignificant with the p-value of 0.46 .

In Fig. 5, the distribution of AIC events within an ICME-driven sheath is further investigated by studying the occurrence rate of AICs as a function of fractional distance $[F]$ from the shock [the blue curves]. The figure also compares the AIC occurrence rate to the one of MMs from Ala-Lahti et al. [2018] [the yellow and orange dashed curves]. The occurrence rate is defined here as the ratio of bins containing AICs to the total number of bins considered, and the curves of different shades of blue indicate the lower limit of AICs we require to be in each bin [ $>0,>2$ or $>7 \mathrm{AICs}]$. The error bars in the light blue curve in Fig. 5 show the relative distribution of AICs within an ICME sheath. Their sum gives 1.0, i.e., indicating all AICs observed in this study. The numerical value of each error bar is given next to it in the figure.

All the curves in Fig. 5, especially the ones with deeper shades of blue, demonstrate that the occurrence rate of AICs is blatantly the highest right after the shock and has a decreasing non-linear trend as a function of $F$ differing from the one of MMs. In addition, the relative distribution given by the error bars decreases monotonically within the fractional distance interval $0<F<0.8$.

We also check that the results are not biased due to the different number of data points included in each sub-region. The near-shock, mid-sheath and near-LE regions contain $280 \mathrm{~h}, 280 \mathrm{~h}$ and $268 \mathrm{~h} \mathrm{[308} \mathrm{h,} 307 \mathrm{~h}, 307 \mathrm{~h}$ ] of data, respectively, and the corresponding occurrence rates of AICs in each sub-region are $0.102,0.052$ and 0.047 [0.089, 0.047, 0.043] when the restrictions of data gaps and the changes in azimuthal and polar directions [the restriction of data gaps] are taken into account. Although the first two sub-regions each have an additional $12 \mathrm{~h}$ of data compared to the near-LE region, the difference only constitutes about $4 \%$ of the total time the spacecraft spent in each sub-region and is largely due to the angular restrictions. In addition, the occurrence rates imply consistency with the results given in Table 2 and Fig. 5 .

\subsection{Statistics of AIC Events}

Figure 6 shows the relative distribution of the ellipticity of an AIC event, $\lambda_{1} / \lambda_{2}$, the ratio of solar wind [SW] speed to the theoretical phase [ph] speed of an AIC event, $V_{s w} / V_{p h}$, and the distributions of the AIC frequencies normalized to the ion cyclotron frequency $\left[\Omega_{i}\right]$ in both the spacecraft and solar wind frames, $\pm \omega_{s c} / \Omega_{i}$ and $\pm \omega_{s w} / \Omega_{i}$, where the sign indicates the polarization.

The ellipticity is distributed throughout the whole interval $1<\lambda_{1} / \lambda_{2}<10$, as illustrated in Fig. 6a. The median and average of the distribution are 4.4 and $4.7 \pm 0.05$. Furthermore, $12 \%$ of AIC events have $\lambda_{1} / \lambda_{2}>8$. 


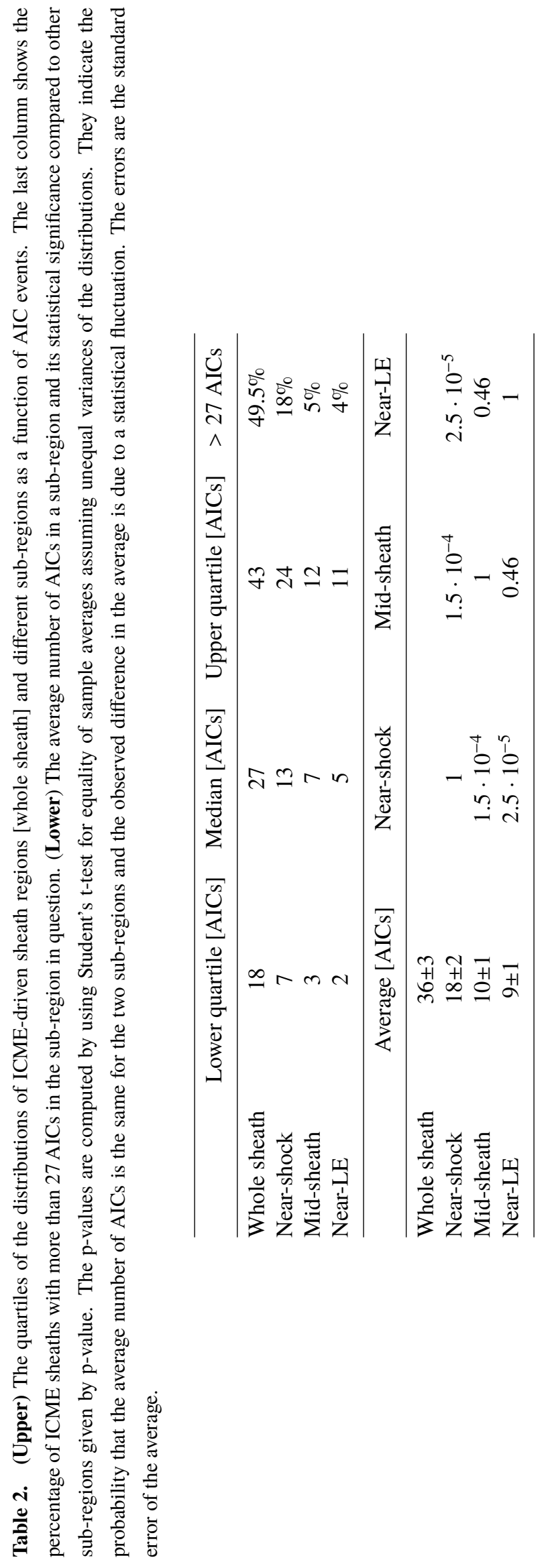


The majority of AIC events have $V_{s w} / V_{p h}>2$, as is evident from Fig. 6b, and there are no events that have the ratio below one. The median and average of the distribution are 4.9 and $7.3 \pm 0.5$, and the interval $3<V_{s w} / V_{p h}<7$ covers $70 \%$ of the distribution implying that the Doppler effect may introduce a considerable shift in the AIC events studied [see Eq. 5].

About half [49\%] of AIC events are RH polarized in the spacecraft frame. However, these events could intrinsically be LH polarized if their propagation is against the solar wind flow. As there is an ambiguity in the absolute propagation direction, all the events with $\mathrm{RH}$ polarization could be $\mathrm{LH}$ in the solar wind frame.

The significance of the Doppler effect in frequency is illustrated in comparison of Fig. $6 \mathrm{c}$ and d. In the spacecraft frame, 72\% of events [RH: 70\%, LH: 73\%] have their frequency below the ion cyclotron frequency whereas the corresponding percentage is $94 \%$ in the solar wind frame [RH: 93\%, LH: 95\%]. Therefore, events having the LH polarization in the SW frame and $\vec{k}$ parallel to the direction of the solar wind flow are Doppler-shifted to higher frequencies maintaining their sense of polarization and could have $-\omega_{s c} / \Omega_{i}<-1$ in the spacecraft frame.

At the end, we investigate AIC events that experience no significant Doppler effect in their frequency and sense of polarization due to their propagation being nearly orthogonal to the solar wind flow [Remya et al., 2014]. Figure 7 shows the distributions of the angle between $\vec{k}$ and $\overrightarrow{V_{s w}}$, $\theta_{k V_{s w}}$ and the term $\frac{V_{s w}}{V_{p h}} \cos \left(\theta_{k V_{s w}}\right)$ in Eq. 5. It can be seen that a notable percentage of AICs have $\theta_{k V_{s w}}>70^{\circ}$ [53\%; Fig. 7a]. However, the overall significance of the Doppler effect is defined by the term $\frac{V_{s w}}{V_{p h}} \cos \left(\theta_{k V_{s w}}\right)$, whose distribution is shown in Fig. 7b. We define $\frac{V_{s w}}{V_{p h}} \cos \left(\theta_{k V_{s w}}\right)=0.25$ as the limit of significance which represents $25 \%$ shift upward in frequency by the Doppler shift and is indicated by a black dashed line in the figure. The AIC events below this boundary cover $10 \%$ of the distribution and are composed of 54\% RH and 46\% LH events, 57\% and $73 \%$ of them having $\omega_{s c} / \Omega_{i}<1$ and $-\omega_{s c} / \Omega_{i}>-1$, respectively.

\subsection{Plasma Parameters within AIC Events}

Figure 8 shows the distribution of AIC events and MMs as a function of ion $\beta_{\|}$and $\beta_{\perp} / \beta_{\|}$. AICs are observed within a wide spread of $\beta_{\|}$and have a notable variation of the anisotropy values, especially in the low $\beta_{\|}$region $\left[\beta_{\|}<1\right]$. However, only $13 \%$ and $7 \%$ of AICs are above the IC and mirror instability curves, respectively. Also MMs are generally below the instability threshold curves. AICs become more infrequent as a function of increasing $\beta_{\|}$whereas majority of MMs are associated with plasma having $\beta_{\|}>2$. This implies that the ratio of the number of observed MMs to the one of observed AICs increases as a function $\beta_{\|}$. Interestingly, the occurrence of MMs approximately starts to increase when the mirror instability threshold curve goes below the IC instability curve. Figure 8 also shows events [1678] omitted due to the shifted FH instability threshold curve.

The plasma conditions associated with AICs, MMs and the whole plasma in ICME sheaths are further investigated in Fig. 9 that shows their ion beta anisotropy distributions. The values of MMs are approximately peaked and confined by $\beta_{\perp} / \beta_{\|}=1 \pm 0.5$ whereas the distributions of AICs and an ICME sheath both have a long tail towards higher values of $\beta_{\perp} / \beta_{\|}$but having the majority [55\% and $59 \%$, respectively] and peaks of the distributions below one.

Table 3 sums up the results of plasma conditions of both wave types and an ICME sheath by giving the averages and median values of each distribution within the entire sheath. In addition, p-values with respect to the averages of AICs are given. AIC events are associated with different ion beta values than MMs. However, median values of $\beta_{\perp}$ and $\beta_{\|}$for AICs do not differ drastically from the values for the whole plasma in an ICME sheath. In addition, the ion beta anisotropy does not show considerable variation between AICs, MMs and the ICME sheath plasma. All the differences of averages given in Table 3 are statistically significant as indicated by the p-values. 


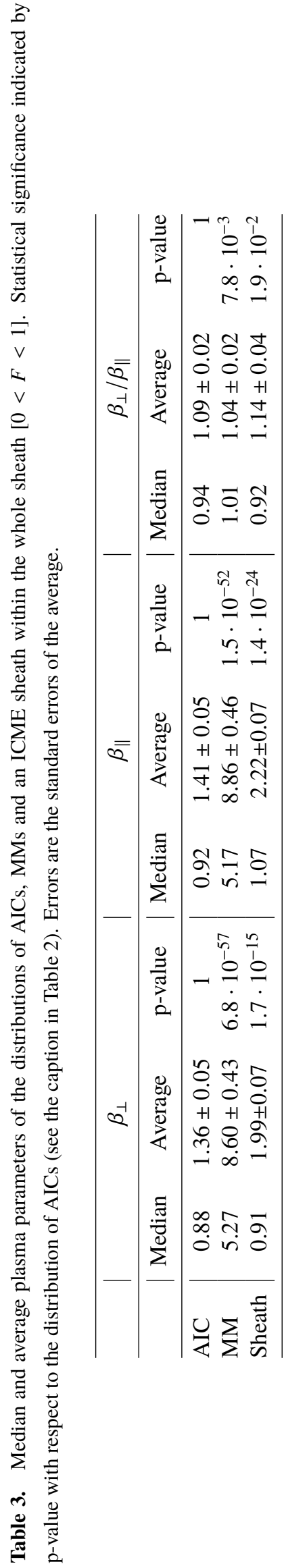

This article is protected by copyright. All rights reserved. 


\section{Conclusion and Discussion}

We have performed a comprehensive statistical study of AIC waves in sheath regions driven by ICMEs. The AIC wave events were identified by constructing an automated identification algorithm and we have investigated their occurrence, distribution within the ICME sheath, properties and plasma surroundings. In addition, we have compared the results to the ones of MMs presented by Ala-Lahti et al. [2018].

The statistical results imply the following key findings:

1. All examined ICME sheath regions had at least two AIC wave events within them.

2. AICs were observed throughout the ICME sheath but the occurrence was weighted towards the vicinity of the shock.

3. In the spacecraft frame, AIC events are equally divided into $\mathrm{LH}$ and $\mathrm{RH}$ polarized cases, and $72 \%$ of the events have their frequency below the IC frequency.

4. In the solar wind frame, $94 \%$ of AICs have their frequency below the IC frequency, and the distribution of $V_{s w} / V_{p h}$ suggests that LH polarized waves might be observed as RH polarized in the spacecraft frame due to the Doppler effect.

5. $87 \%$ of AIC events occurred in stable plasma with respect to the IC instability. AICs are generally associated with lower values of ion $\beta_{\|}$than MMs.

Our results imply that AICs are common structures in ICME-driven sheath regions with the occurrence rate decreasing from the shock to the ejecta leading edge. As the ICME-driven shocks tend to be weak and quasi-perpendicular [e.g., Kilpua et al., 2015; Palmerio et al., 2016; Ala-Lahti et al., 2018], our results are consistent with the findings in the Earth's magnetosheath, where AIC waves are frequently reported [e.g., Schwartz et al., 1996; Remya et al., 2014; Souček et al., 2015]. In the Earth's magnetosheath, AICs are mainly observed in the quasi-perpendicular region of the bow shock during a low Alfvén Mach number conditions [Song et al., 1994; Hubert et al., 1998; Souček et al., 2015], as already mentioned in Section 1. The numerous occurrence of AIC waves in ICME sheaths furthermore supports the suggestion made by Ala-Lahti et al. [2018] that the ICME sheath expansion is analogous to plasma expansion at the flanks of the Earth's magnetosheath, where a notable increase in the occurrence of AICs is also reported [Souček et al., 2015]. On the other hand, AICs are less frequent in the plasma depletion layer [PDL] that occurs in the sub-solar magnetosheath [Anderson and Fuselier, 1993; Souček et al., 2015]. In ICME-driven sheaths, PDL and its formation are less clear [e.g., Dasso et al., 2007; Farrugia et al., 2008; Kilpua et al., 2017a]. It is more likely that in ICME sheaths the decreasing trend of AIC occurrence from the shock to the ejecta leading edge is related to shock-related processes. Moreover, it has been shown in simulations [Shoji et al., 2009] that plasma unstable with respect to the IC instability tend to saturate quickly, and AICs waves damp relatively fast as they are efficient to lose their energy due to nonlinear processes.

Overall, the results of this study and the ones presented by Ala-Lahti et al. [2018] suggest that the shock compression has a crucial role in the process of plasma heating that generates wave activity in sheath regions driven by ICMEs. As mentioned in Section 1, in ICME-driven sheaths, plasma tends to pile in front of an ICME maintaining the record of previous interactions [Siscoe and Odstrcil, 2008]. Thus, they offer a possibility to study the occurrence of different processes taking place at different distances from the Sun. AICs observed closer to the leading edge of the ejecta could be generated in the vicinity of the shock earlier in time. However, the ICME sheath structure might be modified by dynamic processes such as magnetic reconnection, and to construct a deeper understanding on sheath regions driven by ICMEs, we also need direct observations closer to the Sun. We note that field line draping around the driving ejecta in the absence of PDL or alternative phenomena, such as a shock propagating within an ICME sheath [e.g., Lugaz et al., 2015, 2017, and references therein], could also provide sufficient heating that occasionally generates AIC waves in ICME-driven sheath regions.

As the electric field measurements required to determine the absolute direction of the waves are not available, there is an ambiguity in the distribution of the intrinsic polarization [Remya et al., 2014] 
and thus, false positive observations are a possibility. In our analysis, the events that experience insignificant Doppler effect are divided into 46\% LH and 54\% RH polarized events whereas the majority [50/59] of the corresponding events in the study reported by Remya et al. [2014] are LH polarized. However, we point out that only $10 \%$ of AICs observed in this study showed insignificant Doppler shift. Automated identification procedure that investigates data intervals of certain length is naturally capable of reporting false positive events. For the future development, we suggest in-situ studies examining individual AIC waves from the data of a spacecraft that also provides high-resolution electric field measurements, such as European Space Agency's Solar Orbiter [Müller et al., 2013] and NASA's Parker Solar Probe [Fox et al., 2016].

However, we note that the distribution of $V_{s w} / V_{p h}$ suggests that, because of the Doppler shift, intrinsically LH polarized AICs may be observed as RH polarized in the spacecraft frame. In the spacecraft frame, $72 \%$ of the AICs have their frequency below the IC frequency, consistent with the results obtained in the Earth's magnetosheath [Remya et al., 2014]. The distribution of $\theta_{k V_{s w}}$ is similarly consistent with the one reported by Remya et al. [2014]. In addition, the distributions of $\omega_{s c} / \Omega_{i}$ and $\omega_{s w} / \Omega_{i}$ meet the expectations given by the previous work in the solar wind [Jian et al., 2009, 2010]. In agreement with previous observations [e.g., Remya et al., 2014; Yu et al., 2015], we also observed AIC events having a large ellipticity $\left[\lambda_{1} / \lambda_{2}>8\right.$; see also Jian et al., 2014, and references therein].

Finally, we discuss the plasma conditions associated with AICs and MMs in ICME-driven sheath regions. We found that MMs occur in plasmas having distinctly different ion plasma beta values compared to those when AICs are observed. We also note that the conditions during AICs do not considerably differ from those periods when waves are not observed, whereas for MMs the difference is notable. The median and average values of ion beta anisotropy are, on the other hand, comparable and not significantly different for AICs, MMs and the whole ICME sheath plasma [see Table 3]. However, the width of the distribution is different [see Fig. 9] and depends on plasma beta that also regulates the occurrence of instabilities [see Fig. 2 and 8]. This dependence of anisotropy on plasma beta and different values of $\beta_{\|}$associated with AICs, MMs and the ICME sheath are likely the cause of the small variations in the average anisotropy. In addition, we note that there is still an uncertainty of the wave origin in plasma that is stable with respect to both IC and FH instabilities, which can have an effect on the results.

Similarly to models and observations in the Earth's magnetosheath [e.g., Anderson and Fuselier, 1993; Gary et al., 1993; Souček et al., 2015], MMs occupy higher ion $\beta_{\|}$plasma than AICs. Generally, Anderson and Fuselier [1993] and Souček et al. [2015] reported higher ion beta anisotropy values associated with AICs in the Earth's magnetosheath than what we observe in ICME-driven sheaths. However, the anisotropy distribution of AICs has a long tail following the distribution of overall ICME sheath plasma [see Fig. 9]. The different anisotropies may be a consequence of substantial differences between the two plasma environments. The Earth's magnetosheath has generally a stronger bow shock in comparison to interplanetary shocks [e.g., Kilpua et al., 2015; Souček et al., 2015]. ICME sheaths have also characteristics of both expansion and propagation sheaths [Siscoe and Odstrcil, 2008], while planetary magnetosheaths are pure propagation sheaths.

From Figure 8 we can conclude that for a given value of ion $\beta_{\|}$, we are more likely to observe the wave mode which requires a lower anisotropy for the instability to develop. This suggests that comparing to magnetosheath plasma, the relatively weak heating of ICME sheaths typically only drives the plasma to marginal instability where the plasma is stabilized by the instability with a lower threshold. A vast majority of AIC waves as well as mirror modes are thus observed under stable plasma conditions.

In this study, we have used linear theory to predict the stability of plasma. We, however, note that the presence of different ion species, such as $\mathrm{He}^{++}$and $\mathrm{SO}_{2}^{+}$, can modify the possible growth rates of the IC and mirror instabilities [e.g., Gary et al., 1993; Russell et al., 1998; Huddleston et al., 1999]. Furthermore, recent work [e.g., Shaaban et al., 2015, 2016, 2018] has discussed whether the instabilities are sensitive to electron temperature anisotropy and suprathermal particle populations. Shaaban et al. [2016] discovered that the growth rate of the IC instability increases 
in the presence of broad anisotropic suprathermal distribution. Moreover, a lower anisotropy is required to reach the instability threshold if a broad suprathermal population occurs, whereas an electron anisotropy might increase the threshold [Shaaban et al., 2015]. In the case of the mirror instability, suprathermal populations of electrons or protons, and also electron anisotropy decrease the instability threshold. Taking these effects into account in our analysis is beyond the scope of this study. We, however, conclude that investigating the distributions of electron anisotropy and suprathermal particles in ICME-driven sheaths would deepen the understanding achieved by this work and the one by Ala-Lahti et al. [2018].

Finally, we note that the parallel fire hose instability is likely to constrain the plasma anisotropy in ICME-driven sheaths [see Fig. 2 and the omitted events in Fig. 8], consistently with previous results for the solar wind. [e.g., Kasper et al., 2002; Matteini et al., 2006; Zhao et al., 2019].

\section{Acknowledgments}

Data used in this study are available at the NASA Goddard Space Flight Center Coordinated Data Analysis Web [CDAWeb, http://cdaweb.gsfc.nasa.gov/]. The investigated magnetic field data is measured by the Wind Magnetic Fields Investigation instrument. In addition, we use the proton number density, proton velocity vector, and proton thermal speed data, both parallel and perpendicular to the magnetic field, from the Wind Solar Wind Experiment instrument. The Wind data sources and their documentation are given by NASA [https://wind.nasa.gov/data.php]. Furthermore, the studied sheath regions driven by ICMEs are listed by Palmerio et al. [2016] [https://doi.org/10.5194/angeo34-313-2016-supplement].

We thank Adam Szabo for the Wind MFI data and Keith W. Ogilvie for the Wind SWE data. We thank the NASA Goddard Space Flight Center for providing data on CDAWeb. We also thank Palmerio et al. [2016] for the list of sheath regions driven by ICMEs. MAL acknowledges B. Remya and B. T. Tsurutani for the discussion concerning their previous work on AIC waves in the magnetosheath. MAL and EK acknowledge The Finnish Centre of Excellence in Research of Sustainable Space, funded through the Academy of Finland grant number 312351 and Academy of Finland project 1310445. This project has received funding from the European Research Council [ERC] under the European Union's Horizon 2020 research and innovation program [grant agreement no. 724391, SolMAG]. JS acknowledges the support of Czech Science Foundation grant 17-08772S. TP acknowledges funding from the Academy of Finland project 310444. APD acknowledges support from the Swedish Civil Contingencies Agency grant 2016-2102. The authors declare that they have no conflict of interest. 


\section{References}

Ala-Lahti, M. M., E. K. J. Kilpua, A. P. Dimmock, A. Osmane, T. Pulkkinen, and J. Souček (2018), Statistical analysis of mirror mode waves in sheath regions driven by interplanetary coronal mass ejection, Annales Geophysicae, 36, 793-808, doi:10.5194/angeo-36-793-2018.

Anderson, B. J., and S. A. Fuselier (1993), Magnetic pulsations from 0.1 to $4.0 \mathrm{~Hz}$ and associated plasma properties in the earth's subsolar magnetosheath and plasma depletion layer, Journal of Geophysical Research, 98, 1461-1479, doi:10.1029/92JA02197.

Anderson, B. J., S. A. Fuselier, S. P. Gary, and R. E. Denton (1994), Magnetic spectral signatures in the Earth's magnetosheath and plasma depletion layer, Journal of Geophysical Research, 99, 5877-5891, doi:10.1029/93JA02827.

Bale, S. D., J. C. Kasper, G. G. Howes, E. Quataert, C. Salem, and D. Sundkvist (2009), Magnetic Fluctuation Power Near Proton Temperature Anisotropy Instability Thresholds in the Solar Wind, Physical Review Letters, 103(21), 211101, doi:10.1103/PhysRevLett.103.211101.

Blanco-Cano, X., P. Kajdič, E. Aguilar-Rodríguez, C. T. Russell, L. K. Jian, and J. G. Luhmann (2016), Interplanetary shocks and foreshocks observed by STEREO during 2007-2010, Journal of Geophysical Research (Space Physics), 121, 992-1008, doi:10.1002/2015JA021645.

Bothmer, V., and R. Schwenn (1998), The structure and origin of magnetic clouds in the solar wind, Annales Geophysicae, 16, 1-24, doi:10.1007/s00585-997-0001-x.

Burlaga, L., E. Sittler, F. Mariani, and R. Schwenn (1981), Magnetic loop behind an interplanetary shock - Voyager, Helios, and IMP 8 observations, Journal of Geophysical Research, 86, 66736684, doi:10.1029/JA086iA08p06673.

Burlaga, L. F. (1988), Magnetic clouds and force-free fields with constant alpha, Journal of Geophysical Research, 93, 7217-7224, doi:10.1029/JA093iA07p07217.

Chen, J. (2017), Physics of erupting solar flux ropes: Coronal mass ejections (CMEs)- Recent advances in theory and observation, Physics of Plasmas, 24(9), 090501, doi:10.1063/1.4993929.

Crooker, N. U., and G. L. Siscoe (1977), A mechanism for pressure anisotropy and mirror instability in the dayside magnetosheath, Journal of Geophysical Research, 82, 185, doi: 10.1029/JA082i001p00185.

Dasso, S., M. S. Nakwacki, P. Démoulin, and C. H. Mandrini (2007), Progressive Transformation of a Flux Rope to an ICME. Comparative Analysis Using the Direct and Fitted Expansion Methods, Solar Physics, 244, 115-137, doi:10.1007/s11207-007-9034-2.

Davidson, R. C., and J. M. Ogden (1975), Electromagnetic ion cyclotron instability driven by ion energy anisotropy in high-beta plasmas, Physics of Fluids, 18, 1045-1050, doi:10.1063/1.861253.

Démoulin, P., S. Dasso, and M. Janvier (2018), Exploring the biases of a new method based on minimum variance for interplanetary magnetic clouds, Astronomy \& Astrophysics, 619, A139, doi:10.1051/0004-6361/201833831.

Dimmock, A. P., A. Osmane, T. I. Pulkkinen, and K. Nykyri (2015), A statistical study of the dawndusk asymmetry of ion temperature anisotropy and mirror mode occurrence in the terrestrial dayside magnetosheath using THEMIS data, Journal of Geophysical Research (Space Physics), 120, 5489-5503, doi:10.1002/2015JA021192.

Farrugia, C. J., N. V. Erkaev, U. Taubenschuss, V. A. Shaidurov, C. W. Smith, and H. K. Biernat (2008), A slow mode transition region adjoining the front boundary of a magnetic cloud as a relic of a convected solar wind feature: Observations and MHD simulation, Journal of Geophysical Research (Space Physics), 113, A00B01, doi:10.1029/2007JA012953.

Feng, H., and J. Wang (2013), Magnetic-reconnection exhausts in the sheath of magnetic clouds, Astronomy \& Astrophysics, 559, A92, doi:10.1051/0004-6361/201322522.

Fowler, R. A., B. J. Kotick, and R. D. Elliott (1967), Polarization analysis of natural and artificially induced geomagnetic micropulsations, Journal of Geophysical Research, 72(11), 2871-2883, doi:10.1029/JZ072i011p02871.

Fox, N. J., M. C. Velli, S. D. Bale, R. Decker, A. Driesman, R. A. Howard, J. C. Kasper, J. Kinnison, M. Kusterer, D. Lario, M. K. Lockwood, D. J. McComas, N. E. Raouafi, and A. Szabo (2016), The Solar Probe Plus Mission: Humanity's First Visit to Our Star, Space Science Reviews, 204, 7-48, doi:10.1007/s11214-015-0211-6. 
Fuselier, S. A., B. J. Anderson, S. P. Gary, and R. E. Denton (1994), Inverse correlations between the ion temperature anisotropy and plasma beta in the Earth's quasi-parallel magnetosheath, Journal of Geophysical Research, 99, 14,931-14,936, doi:10.1029/94JA00865.

Gary, S. P. (1992), The mirror and ion cyclotron anisotropy instabilities, Journal of Geophysical Research, 97, 8519-8529, doi:10.1029/92JA00299.

Gary, S. P., S. A. Fuselier, and B. J. Anderson (1993), Ion anisotropy instabilities in the magnetosheath, Journal of Geophysical Research, 98, 1481-1488, doi:10.1029/92JA01844.

Gary, S. P., H. Li, S. O'Rourke, and D. Winske (1998), Proton resonant firehose instability: Temperature anisotropy and fluctuating field constraints, Journal of Geophysical Research, 103, 14,56714,574, doi:10.1029/98JA01174.

Gary, S. P., L. K. Jian, T. W. Broiles, M. L. Stevens, J. J. Podesta, and J. C. Kasper (2016), Ion-driven instabilities in the solar wind: Wind observations of 19 March 2005, Journal of Geophysical Research (Space Physics), 121, 30-41, doi:10.1002/2015JA021935.

Génot, V., E. Budnik, C. Jacquey, I. Dandouras, and E. Lucek (2009), Mirror Modes Observed with Cluster in the Earth's Magnetosheath: Statistical Study and IMF/Solar Wind Dependence, Advances in Geosciences, Volume 14: Solar Terrestrial (ST), 14, 263-283, doi: 10.1142/9789812836205_0019.

Good, S. W., R. J. Forsyth, J. P. Eastwood, and C. Möstl (2018), Correlation of ICME Magnetic Fields at Radially Aligned Spacecraft, Solar Physics, 293, 52, doi:10.1007/s11207-018-1264-y.

Gosling, J. T., and D. J. McComas (1987), Field line draping about fast coronal mass ejecta - A source of strong out-of-the-ecliptic interplanetary magnetic fields, Geophysical Research Letters, 14, 355-358, doi:10.1029/GL014i004p00355.

Guo, X., L. Sironi, and R. Narayan (2017), Electron Heating in Low-Mach-number Perpendicular Shocks. I. Heating Mechanism, The Astrophysical Journal, 851, 134, doi:10.3847/1538-4357/ aa9b82.

Hasegawa, A. (1969), Drift mirror instability of the magnetosphere., Physics of Fluids, 12, 2642 2650, doi:10.1063/1.1692407.

Hellinger, P. (2007), Comment on the linear mirror instability near the threshold, Physics of Plasmas, 14(8), 082105, doi:10.1063/1.2768318.

Hellinger, P., P. Trávníček, A. Mangeney, and R. Grappin (2003), Hybrid simulations of the magnetosheath compression: Marginal stability path, Geophysical Research Letter, 30, 1959, doi: 10.1029/2003GL017855.

Hellinger, P., P. Trávníček, J. C. Kasper, and A. J. Lazarus (2006), Solar wind proton temperature anisotropy: Linear theory and WIND/SWE observations, Geophysical Research Letters, 33, L09101, doi:10.1029/2006GL025925.

Hubert, D., C. Lacombe, C. C. Harvey, M. Moncuquet, C. T. Russell, and M. F. Thomsen (1998), Nature, properties, and origin of low-frequency waves from an oblique shock to the inner magnetosheath, Journal of Geophysical Research, 103, 26,783-26,798, doi:10.1029/98JA01011.

Huddleston, D. E., R. J. Strangeway, X. Blanco-Cano, C. T. Russell, M. G. Kivelson, and K. K. Khurana (1999), Mirror-mode structures at the Galileo-Io flyby: Instability criterion and dispersion analysis, Journal of Geophysical Research, 104, 17,479-17,490, doi:10.1029/1999JA900195.

Huttunen, K. E. J., H. E. J. Koskinen, and R. Schwenn (2002), Variability of magnetospheric storms driven by different solar wind perturbations, Journal of Geophysical Research (Space Physics), 107, 1121, doi:10.1029/2001JA900171.

Isavnin, A., A. Vourlidas, and E. K. J. Kilpua (2014), Three-Dimensional Evolution of Flux-Rope CMEs and Its Relation to the Local Orientation of the Heliospheric Current Sheet, Solar Physics, 289, 2141-2156, doi:10.1007/s11207-013-0468-4.

Jian, L. K., C. T. Russell, J. G. Luhmann, R. J. Strangeway, J. S. Leisner, and A. B. Galvin (2009), Ion Cyclotron Waves in the Solar Wind Observed by STEREO Near 1 AU, The Astrophysical Journal Letters, 701, L105-L109, doi:10.1088/0004-637X/701/2/L105.

Jian, L. K., C. T. Russell, J. G. Luhmann, B. J. Anderson, S. A. Boardsen, R. J. Strangeway, M. M. Cowee, and A. Wennmacher (2010), Observations of ion cyclotron waves in the solar wind near 0.3 AU, Journal of Geophysical Research (Space Physics), 115, A12115, doi:10.1029/2010JA015737. 
Jian, L. K., H. Y. Wei, C. T. Russell, J. G. Luhmann, B. Klecker, N. Omidi, P. A. Isenberg, M. L. Goldstein, A. Figueroa-Viñas, and X. Blanco-Cano (2014), Electromagnetic Waves near the Proton Cyclotron Frequency: STEREO Observations, The Astrophysical Journal, 786, 123, doi:10.1088/0004-637X/786/2/123.

Kajdič, P., X. Blanco-Cano, E. Aguilar-Rodriguez, C. T. Russell, L. K. Jian, and J. G. Luhmann (2012), Waves upstream and downstream of interplanetary shocks driven by coronal mass ejections, Journal of Geophysical Research (Space Physics), 117, A06103, doi:10.1029/2011JA017381.

Kasper, J. C., A. J. Lazarus, and S. P. Gary (2002), Wind/SWE observations of firehose constraint on solar wind proton temperature anisotropy, Geophysical Research Letters, 29, 1839, doi:10.1029/ 2002GL015128.

Kataoka, R., D. Shiota, E. Kilpua, and K. Keika (2015), Pileup accident hypothesis of magnetic storm on 17 March 2015, Geophysical Research Letters, 42, 5155-5161, doi:10.1002/2015GL064816.

Kaufmann, R. L., J.-T. Horng, and A. Wolfe (1970), Large-amplitude hydromagnetic waves in the inner magnetosheath, Journal of Geophysical Research, 75, 4666, doi:10.1029/JA075i025p04666.

Kaymaz, Z., and G. Siscoe (2006), Field-Line Draping Around ICMES, Solar Physics, 239, 437-448, doi:10.1007/s11207-006-0308-x.

Kilpua, E., H. E. J. Koskinen, and T. I. Pulkkinen (2017a), Coronal mass ejections and their sheath regions in interplanetary space, Living Reviews in Solar Physics, 14, 5, doi:10.1007/ s41116-017-0009-6.

Kilpua, E. K. J., E. Lumme, K. Andreeova, A. Isavnin, and H. E. J. Koskinen (2015), Properties and drivers of fast interplanetary shocks near the orbit of the Earth (1995-2013), Journal of Geophysical Research (Space Physics), 120, 4112-4125, doi:10.1002/2015JA021138.

Kilpua, E. K. J., A. Balogh, R. von Steiger, and Y. D. Liu (2017b), Geoeffective Properties of Solar Transients and Stream Interaction Regions, Space Science Reviews, 212, 1271-1314, doi: 10.1007/s11214-017-0411-3.

Lepping, R. P., M. H. Acũna, L. F. Burlaga, W. M. Farrell, J. A. Slavin, K. H. Schatten, F. Mariani, N. F. Ness, F. M. Neubauer, Y. C. Whang, J. B. Byrnes, R. S. Kennon, P. V. Panetta, J. Scheifele, and E. M. Worley (1995), The Wind Magnetic Field Investigation, Space Science Reviews, 71, 207-229, doi:10.1007/BF00751330.

Liu, Y., J. D. Richardson, J. W. Belcher, J. C. Kasper, and R. M. Skoug (2006), Plasma depletion and mirror waves ahead of interplanetary coronal mass ejections, Journal of Geophysical Research (Space Physics), 111, A09108, doi:10.1029/2006JA011723.

Lugaz, N., C. J. Farrugia, C. W. Smith, and K. Paulson (2015), Shocks inside CMEs: A survey of properties from 1997 to 2006, Journal of Geophysical Research (Space Physics), 120, 2409-2427, doi:10.1002/2014JA020848.

Lugaz, N., C. J. Farrugia, R. M. Winslow, N. Al-Haddad, E. K. J. Kilpua, and P. Riley (2016), Factors affecting the geoeffectiveness of shocks and sheaths at $1 \mathrm{AU}$, Journal of Geophysical Research (Space Physics), 121, 10, doi:10.1002/2016JA023100.

Lugaz, N., M. Temmer, Y. Wang, and C. J. Farrugia (2017), The Interaction of Successive Coronal Mass Ejections: A Review, Solar Physics, 292, 64, doi:10.1007/s11207-017-1091-6.

Matteini, L., S. Landi, P. Hellinger, and M. Velli (2006), Parallel proton fire hose instability in the expanding solar wind: Hybrid simulations, Journal of Geophysical Research (Space Physics), 111(A10), A10101, doi:10.1029/2006JA011667.

Means, J. D. (1972), Use of the three-dimensional covariance matrix in analyzing the polarization properties of plane waves, Journal of Geophysical Research, 77, 5551-5559, doi: 10.1029/JA077i028p05551.

Möstl, C., C. J. Farrugia, E. K. J. Kilpua, L. K. Jian, Y. Liu, J. P. Eastwood, R. A. Harrison, D. F. Webb, M. Temmer, D. Odstrcil, J. A. Davies, T. Rollett, J. G. Luhmann, N. Nitta, T. Mulligan, E. A. Jensen, R. Forsyth, B. Lavraud, C. A. de Koning, A. M. Veronig, A. B. Galvin, T. L. Zhang, and B. J. Anderson (2012), Multi-point Shock and Flux Rope Analysis of Multiple Interplanetary Coronal Mass Ejections around 2010 August 1 in the Inner Heliosphere, The Astrophysical Journal, 758, 10, doi:10.1088/0004-637X/758/1/10.

Müller, D., R. G. Marsden, O. C. St. Cyr, and H. R. Gilbert (2013), Solar Orbiter . Exploring the Sun-Heliosphere Connection, Solar Physics, 285, 25-70, doi:10.1007/s11207-012-0085-7. 
Nieves-Chinchilla, T., M. G. Linton, M. A. Hidalgo, A. Vourlidas, N. P. Savani, A. Szabo, C. Farrugia, and W. Yu (2016), A Circular-cylindrical Flux-rope Analytical Model for Magnetic Clouds, The Astrophysical Journal, 823, 27, doi:10.3847/0004-637X/823/1/27.

Nieves-Chinchilla, T., M. G. Linton, M. A. Hidalgo, and A. Vourlidas (2018), Elliptic-cylindrical Analytical Flux Rope Model for Magnetic Clouds, The Astrophysical Journal, 861, 139, doi: 10.3847/1538-4357/aac951.

Ogilvie, K. W., D. J. Chornay, R. J. Fritzenreiter, F. Hunsaker, J. Keller, J. Lobell, G. Miller, J. D. Scudder, E. C. Sittler, Jr., R. B. Torbert, D. Bodet, G. Needell, A. J. Lazarus, J. T. Steinberg, J. H. Tappan, A. Mavretic, and E. Gergin (1995), SWE, A Comprehensive Plasma Instrument for the Wind Spacecraft, Space Science Reviews, 71, 55-77, doi:10.1007/BF00751326.

Osmane, A., A. P. Dimmock, and T. I. Pulkkinen (2015), Universal properties of mirror mode turbulence in the Earth's magnetosheath, Geophysical Research Letters, 42, 3085-3092, doi: 10.1002/2015GL063771.

Palmerio, E., E. K. J. Kilpua, and N. P. Savani (2016), Planar magnetic structures in coronal mass ejection-driven sheath regions, Annales Geophysicae, 34, 313-322, doi:10.5194/ angeo-34-313-2016.

Palmerio, E., E. K. J. Kilpua, C. Möstl, V. Bothmer, A. W. James, L. M. Green, A. Isavnin, J. A. Davies, and R. A. Harrison (2018), Coronal Magnetic Structure of Earthbound CMEs and In Situ Comparison, Space Weather, 16, 442-460, doi:10.1002/2017SW001767.

Quest, K. B., and V. D. Shapiro (1996), Evolution of the fire-hose instability: Linear theory and wavewave coupling, Journal of Geophysical Research, 101, 24,457-24,470, doi:10.1029/96JA01534.

Rankin, D., and R. Kurtz (1970), Statistical study of micropulsation polarizations, Journal of Geophysical Research, 75, 5444-5458, doi:10.1029/JA075i028p05444.

Remya, B., R. V. Reddy, B. T. Tsurutani, G. S. Lakhina, and E. Echer (2013), Ion temperature anisotropy instabilities in planetary magnetosheaths, Journal of Geophysical Research (Space Physics), 118, 785-793, doi:10.1002/jgra.50091.

Remya, B., B. T. Tsurutani, R. V. Reddy, G. S. Lakhina, B. J. Falkowski, E. Echer, and K.-H. Glassmeier (2014), Large-amplitude, Circularly Polarized, Compressive, Obliquely Propagating Electromagnetic Proton Cyclotron Waves Throughout the Earth's Magnetosheath: Low Plasma $\beta$ Conditions, The Astrophysical Journal, 793, 6, doi:10.1088/0004-637X/793/1/6.

Remya, B., B. T. Tsurutani, R. V. Reddy, G. S. Lakhina, and R. Hajra (2015), Electromagnetic cyclotron waves in the dayside subsolar outer magnetosphere generated by enhanced solar wind pressure: EMIC wave coherency, Journal of Geophysical Research (Space Physics), 120, 75367551, doi:10.1002/2015JA021327.

Ruffenach, A., B. Lavraud, C. J. Farrugia, P. Démoulin, S. Dasso, M. J. Owens, J.-A. Sauvaud, A. P. Rouillard, A. Lynnyk, C. Foullon, N. P. Savani, J. G. Luhmann, and A. B. Galvin (2015), Statistical study of magnetic cloud erosion by magnetic reconnection, Journal of Geophysical Research (Space Physics), 120, 43-60, doi:10.1002/2014JA020628.

Russell, C. T., M. G. Kivelson, K. K. Khurana, and D. E. Huddleston (1998), Magnetic fluctuations close to Io: ion cyclotron and mirror mode wave properties, Planetary and Space Science, 47, 143-150, doi:10.1016/S0032-0633(98)00090-7.

Ruxton, G. D. (2006), The unequal variance t-test is an underused alternative to student's t-test and the mann-whitney u test, Behavioral Ecology, 17(4), 688-690, doi:10.1093/beheco/ark016.

Schwartz, S. J., D. Burgess, and J. J. Moses (1996), Low-frequency waves in the Earthś magnetosheath: present status, Annales Geophysicae, 14, 1134-1150, doi:10.1007/s00585-996-1134-z.

Shaaban, S. M., M. Lazar, S. Poedts, and A. Elhanbaly (2015), Effects of Electrons on the Electromagnetic Ion Cyclotron Instability: Solar Wind Implications, The Astrophysical Journal, 814, 34, doi:10.1088/0004-637X/814/1/34.

Shaaban, S. M., M. Lazar, S. Poedts, and A. Elhanbaly (2016), The interplay of the solar wind proton core and halo populations: EMIC instability, Journal of Geophysical Research (Space Physics), 121, 6031-6047, doi:10.1002/2016JA022587.

Shaaban, S. M., M. Lazar, P. Astfalk, and S. Poedts (2018), Stimulated Mirror Instability From the Interplay of Anisotropic Protons and Electrons, and their Suprathermal Populations, Journal of Geophysical Research (Space Physics), 123, 1754-1766, doi:10.1002/2017JA025066. 
Shoji, M., Y. Omura, B. T. Tsurutani, O. P. Verkhoglyadova, and B. Lembege (2009), Mirror instability and L-mode electromagnetic ion cyclotron instability: Competition in the Earth's magnetosheath, Journal of Geophysical Research (Space Physics), 114, A10203, doi:10.1029/ 2008JA014038.

Shoji, M., Y. Omura, and L.-C. Lee (2012), Multidimensional nonlinear mirror-mode structures in the Earth's magnetosheath, Journal of Geophysical Research (Space Physics), 117, A08208, doi:10.1029/2011JA017420.

Siscoe, G., and D. Odstrcil (2008), Ways in which ICME sheaths differ from magnetosheaths, Journal of Geophysical Research (Space Physics), 113, A00B07, doi:10.1029/2008JA013142.

Siscoe, G., P. J. MacNeice, and D. Odstrcil (2007), East-west asymmetry in coronal mass ejection geoeffectiveness, Space Weather, 5, S04002, doi:10.1029/2006SW000286.

Siu-Tapia, A., X. Blanco-Cano, P. Kajdic, E. Aguilar-Rodriguez, C. T. Russell, L. K. Jian, and J. G. Luhmann (2015), Low-frequency waves within isolated magnetic clouds and complex structures: STEREO observations, Journal of Geophysical Research (Space Physics), 120, 2363-2381, doi: 10.1002/2014JA020568.

Song, P., C. T. Russell, and S. P. Gary (1994), Identification of low-frequency fluctuations in the terrestrial magnetosheath, Journal of Geophysical Research, 99, 6011-6025, doi:10.1029/ 93JA03300.

Sonnerup, B. U. O., and L. J. Cahill, Jr. (1967), Magnetopause Structure and Attitude from Explorer 12 Observations, Journal of Geophysical Research, 72, 171, doi:10.1029/JZ072i001p00171.

Souček, J., E. Lucek, and I. Dandouras (2008), Properties of magnetosheath mirror modes observed by Cluster and their response to changes in plasma parameters, Journal of Geophysical Research (Space Physics), 113, A04203, doi:10.1029/2007JA012649.

Souček, J., C. P. Escoubet, and B. Grison (2015), Magnetosheath plasma stability and ULF wave occurrence as a function of location in the magnetosheath and upstream bow shock parameters, Journal of Geophysical Research (Space Physics), 120, 2838-2850, doi:10.1002/2015JA021087.

Tsurutani, B. T., E. J. Smith, and D. E. Jones (1983), Waves observed upstream of interplanetary shocks, Journal of Geophysical Research, 88, 5645-5656, doi:10.1029/JA088iA07p05645.

Tsurutani, B. T., W. D. Gonzalez, F. Tang, S. I. Akasofu, and E. J. Smith (1988), Origin of interplanetary southward magnetic fields responsible for major magnetic storms near solar maximum (1978-1979), Journal of Geophysical Research, 93, 8519-8531, doi:10.1029/JA093iA08p08519.

Tsurutani, B. T., E. Echer, I. Richter, C. Koenders, and K.-H. Glassmeier (2013), SLAMS at comet 19P/Borrelly: DS1 observations, Planetary and Space Science, 75, 17-27, doi:10.1016/j.pss. 2012.11.002.

Verkhoglyadova, O. P., B. T. Tsurutani, and G. S. Lakhina (2010), Properties of obliquely propagating chorus, Journal of Geophysical Research (Space Physics), 115, A00F19, doi: 10.1029/2009JA014809.

Webb, D. F., and T. A. Howard (2012), Coronal Mass Ejections: Observations, Living Reviews in Solar Physics, 9, 3, doi:10.12942/lrsp-2012-3.

Weibel, E. S. (1970), Ion Cyclotron Instability, Physics of Fluids, 13, 3003-3006, doi:10.1063/1. 1692893.

Welch, B. L. (1938), The significance of the difference between two means when the population variances are unequal, Biometrika, 29(3/4), 350-362, doi:10.2307/2332010.

Wicks, R. T., R. L. Alexander, M. Stevens, L. B. Wilson, III, P. S. Moya, A. Viñas, L. K. Jian, D. A. Roberts, S. O'Modhrain, J. A. Gilbert, and T. H. Zurbuchen (2016), A Proton-cyclotron Wave Storm Generated by Unstable Proton Distribution Functions in the Solar Wind, The Astrophysical Journal, 819, 6, doi:10.3847/0004-637X/819/1/6.

Yu, X., Z. Yuan, D. Wang, H. Li, S. Huang, Z. Wang, Q. Zheng, M. Zhou, C. A. Kletzing, and J. R. Wygant (2015), In situ observations of EMIC waves in $\mathrm{O}^{+}$band by the Van Allen Probe A, Geophysical Research Letters, 42, 1312-1317, doi:10.1002/2015GL063250.

Zhang, J., I. G. Richardson, D. F. Webb, N. Gopalswamy, E. Huttunen, J. C. Kasper, N. V. Nitta, W. Poomvises, B. J. Thompson, C.-C. Wu, S. Yashiro, and A. N. Zhukov (2007), Solar and interplanetary sources of major geomagnetic storms (Dst $=-100 \mathrm{nT}$ ) during 1996-2005, Journal of Geophysical Research (Space Physics), 112, A10102, doi:10.1029/2007JA012321. 
Zhang, T. L., C. T. Russell, W. Baumjohann, L. K. Jian, M. A. Balikhin, J. B. Cao, C. Wang, X. BlancoCano, K.-H. Glassmeier, W. Zambelli, M. Volwerk, M. Delva, and Z. Vörös (2008), Characteristic size and shape of the mirror mode structures in the solar wind at $0.72 \mathrm{AU}$, Geophysical Research Letters, 35, L10106, doi:10.1029/2008GL033793.

Zhang, T. L., W. Baumjohann, C. T. Russell, L. K. Jian, C. Wang, J. B. Cao, M. Balikhin, X. BlancoCano, M. Delva, and M. Volwerk (2009), Mirror mode structures in the solar wind at $0.72 \mathrm{AU}$, Journal of Geophysical Research (Space Physics), 114, A10107, doi:10.1029/2009JA014103.

Zhao, G. Q., H. Q. Feng, D. J. Wu, Y. H. Chu, and J. Huang (2017), Time-dependent Occurrence Rate of Electromagnetic Cyclotron Waves in the Solar Wind: Evidence for the Effect of Alpha Particles?, The Astrophysical Journal Letters, 847, L8, doi:10.3847/2041-8213/aa88b3.

Zhao, G. Q., H. Q. Feng, D. J. Wu, G. Pi, and J. Huang (2019), On the Generation Mechanism of Electromagnetic Cyclotron Waves in the Solar Wind: Statistical Results from Wind Observations, The Astrophysical Journal, 871, 175, doi:10.3847/1538-4357/aaf8b8. 
Figure 1. Relative frequency of (a) $\theta_{k B}$ and $\lambda_{1} / \lambda_{2}$, (b) $\delta B_{\perp}^{2} / \bar{B}^{2}$ and $\delta B_{\perp}^{2} / \delta B_{\|}^{2}$ in sheath regions driven by ICMEs. The black vertical and horizontal lines mark the limits of the criteria used in the AIC event identification [see Eq. 3]. Note that there is an order of magnitude difference between the color scales.

Figure 2. Relative frequency of plasma conditions in sheath regions driven by ICMEs according to ion beta anisotropy $\left[\beta_{\perp} / \beta_{\|}\right]$and parallel ion beta $\left[\beta_{\|}\right]$. Black and yellow overplotted curves indicate the instability thresholds for ion cyclotron and mirror instabilities. Dark purple curve show parallel fire hose $[\mathrm{FH}]$ instability threshold. Light purple curve, below which identified events are omitted, indicates the FH instability threshold with a $33 \%$ shift with respect to the normal direction. The figure shows $99.4 \%$ of the investigated ICME sheath plasma.

Figure 3. Examples of identified AIC events in an ICME-driven sheath region. The shock preceding the ICME passed the Wind spacecraft at 00:52 UT on 10 January 1997. Axes are the maximum $\left[B_{1}\right]$ and intermediate $\left[B_{2}\right]$ variance components and time. The color scale from bright yellow to dark blue indicates progress in time.

Figure 4. Frequency histogram of sheath regions driven by ICMEs according to (a) the occurrence rate of AICs in bins of 0.01 and (b) the number of AIC events in bins of 5 events. The black dashed lines show the lower [(a) 0.03, (b) $18 \mathrm{AICs}]$, median [(a) 0.05, (b) $27 \mathrm{AICs}]$ and upper [(a) 0.07, (b) $43 \mathrm{AICs}]$ quartiles of the distributions.

Figure 5. Occurrence rate of AICs and MMs as a function of $F$ from the ICME-driven shock in bins of $0.1[F=0$ refers to the shock and $F=1$ to the ICME leading edge]. Different shades of blue show different requirements for the number of AIC events in a bin. Yellow and orange dashed curves show the occurrence rate of MMs given by Ala-Lahti et al. [2018]. The occurrence rate is defined as the ratio of the number of AIC events to the total number of intervals within each bin [91 for all intervals]. The error bars of the curve of light blue represent the relative division of AIC events within the whole ICME sheath and are defined as the ratio of the number of AIC events observed within each bin to the total number of AIC events within whole $F$ interval from 0 to 1 . The percentage of AICs in each bin is given next to the error bars.

Figure 6. Relative frequency distribution of (a) the ellipticity of AIC events given by $\lambda_{1} / \lambda_{2}$ in bins of 0.5 , (b) the ratio of the solar wind speed to the phase speed of an AIC event, $V_{s w} / V_{p h}$, in bins of 0.5 , (c) the ratio of the angular frequency of an AIC event in the spacecraft frame to the ion cyclotron frequency, $\omega_{s c} / \Omega_{i}$, in bins of 0.2, and (d) the ratio of the Doppler shifted angular frequency of an AIC event in the solar wind frame to the ion cyclotron frequency, $\omega_{s w} / \Omega_{i}$, in bins of 0.2. The plus [minus] sign of $\omega_{s c} / \Omega_{i}$ indicates the observed right-handed [left-handed] polarization in panel (c) and is also given for $\omega_{s w} / \Omega_{i}$ in panel (d).

Figure 7. Relative frequency distribution of (a) the angle between propagation direction of an AIC event and the solar wind flow, $\theta_{k V_{s w}}$, in bins of $5^{\circ}$ and (b) the term $\frac{V_{s w}}{V_{p h}} \cos \left(\theta_{k V_{s w}}\right)$ given in the Doppler shift relation in Eq. 5 in bins of 0.05 . (b) The black dashed line shows $\frac{V_{s w}}{V_{p h}} \cos \left(\theta_{k V_{s w}}\right)=0.25$, the limit of significance for the Doppler effect.

Figure 8. Distribution of AIC events [blue dots], MMs [orange dots] and omitted events [white dots] in the space of ion beta anisotropy $\left[\beta_{\perp} / \beta_{\|}\right]$and parallel ion beta $\left[\beta_{\|}\right]$. Black and yellow overplotted curves indicate the instability thresholds for ion cyclotron and mirror instabilities, respectively, whereas dark purple curve show FH instability threshold. Light purple curve, below which identified events are omitted, indicates the FH instability threshold with a $33 \%$ shift with respect to the normal direction. The figure shows $99.0 \%$ of the identified AIC events. 
Figure 9. Relative frequency of ion beta anisotropy $\left[\beta_{\perp} / \beta_{\|}\right]$in the surroundings of AIC events [orange curve] and MMs [yellow curve; Ala-Lahti et al., 2018], and generally in ICME-driven sheath regions [solid black curve]. The dashed black vertical line shows the boundary $\beta_{\perp} / \beta_{\|}=1$. 

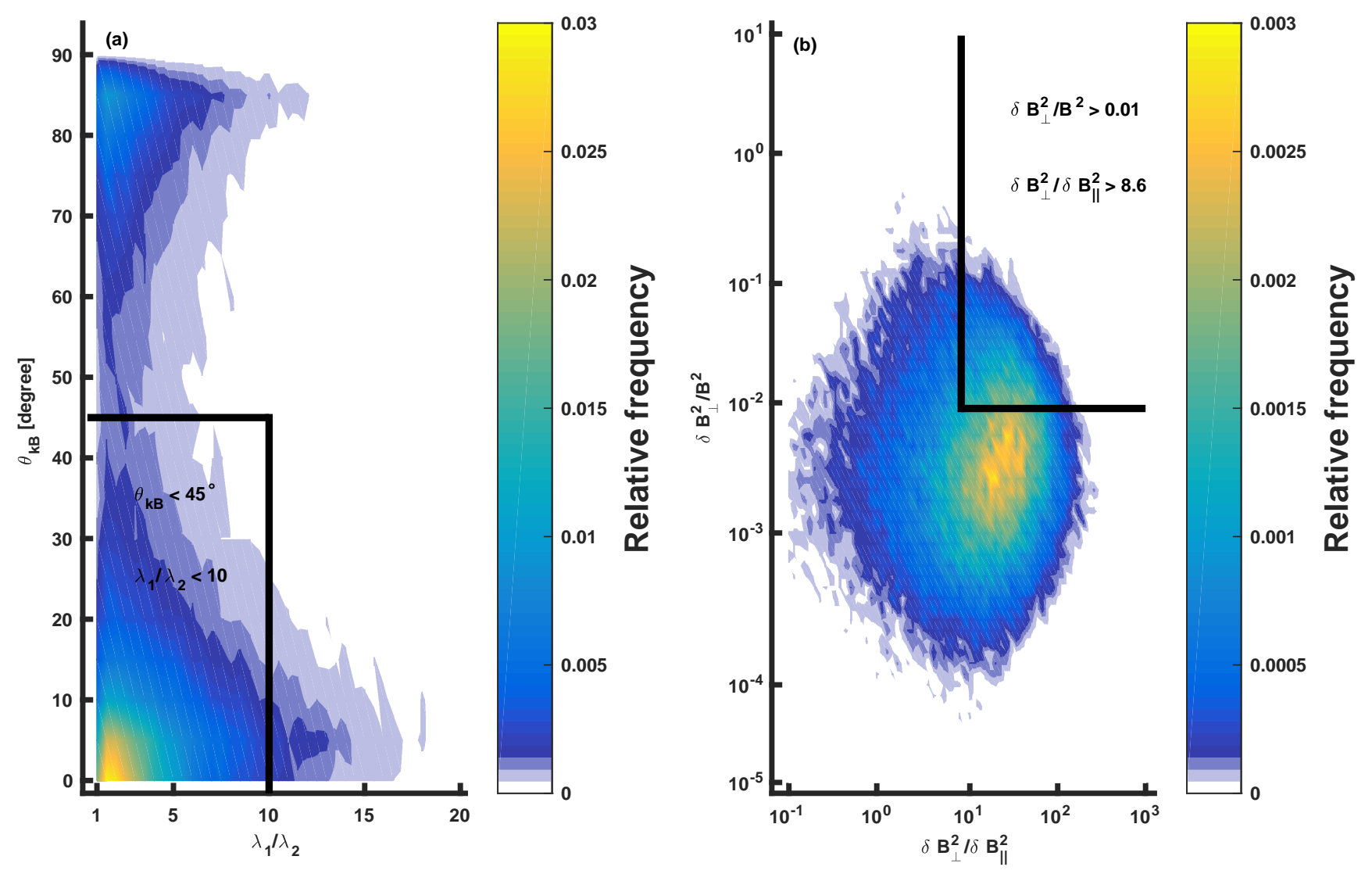
Figure 2.
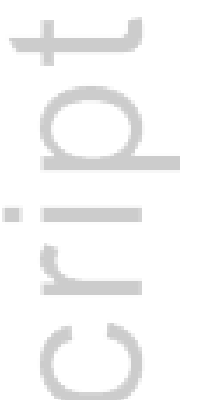

$\infty$
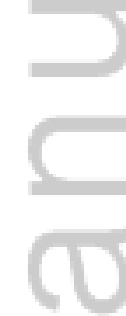

This article is protected by copyright. All rights reserved. 


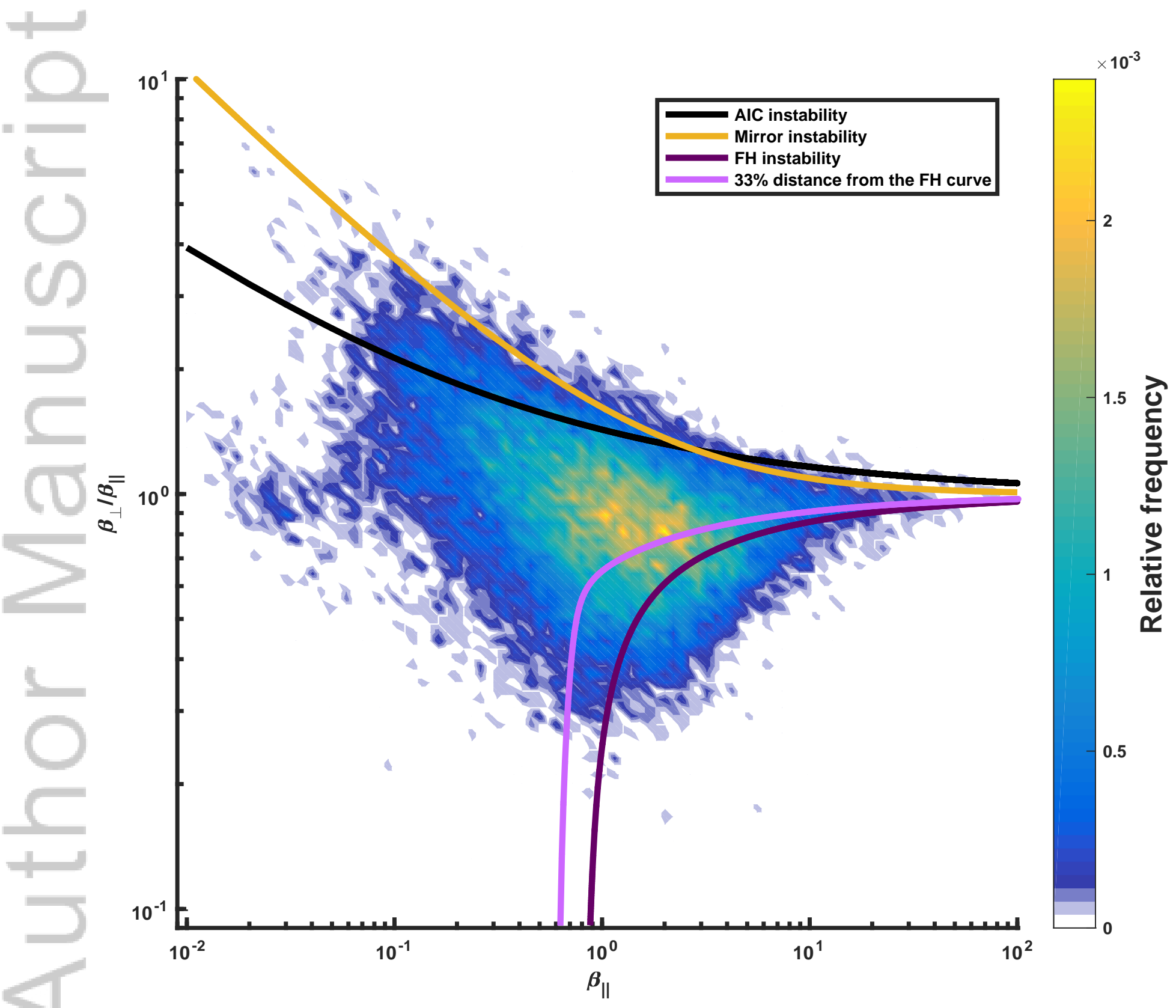

This article is protected by copyright. All rights reserved. 

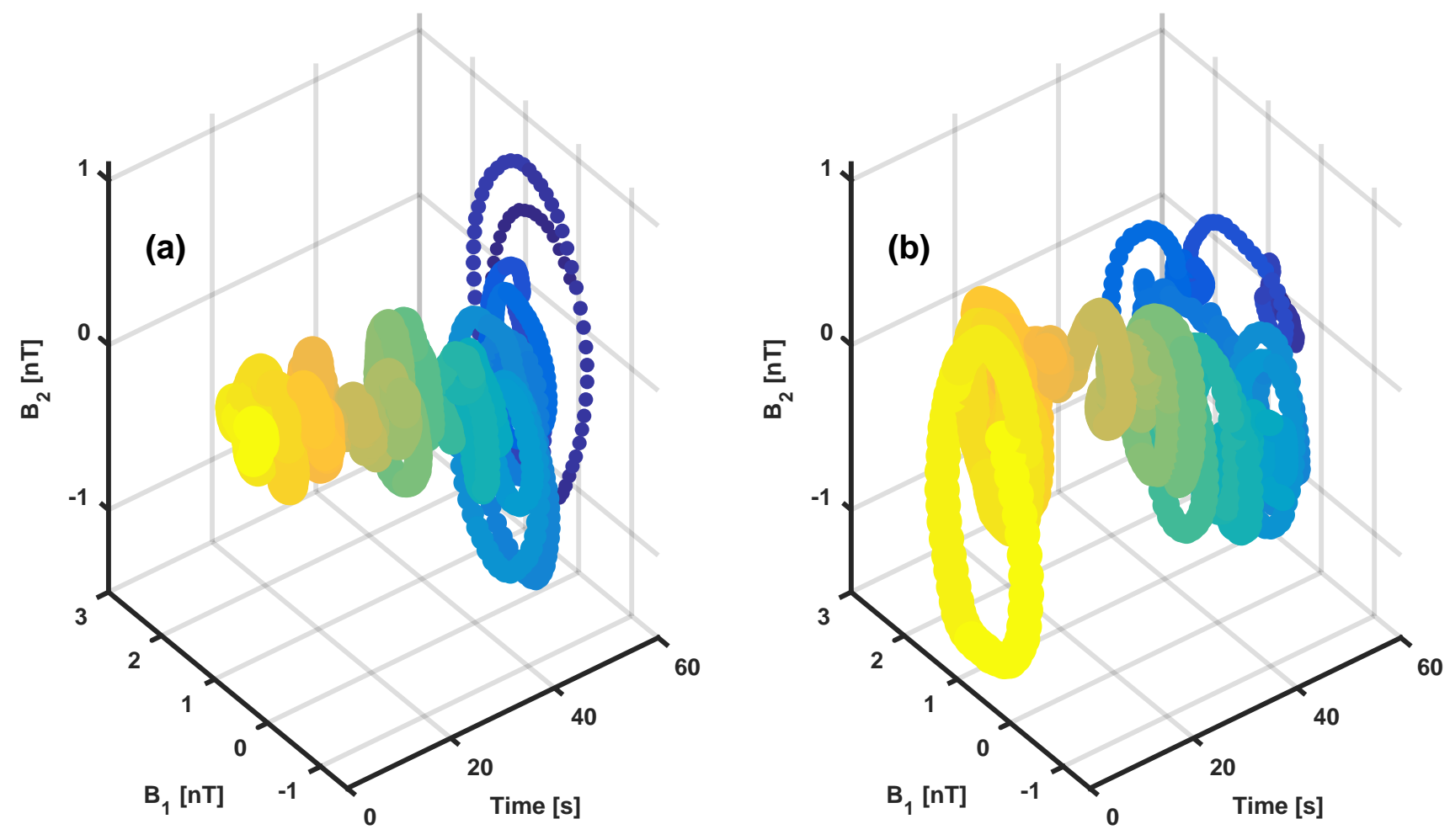

This article is protected by copyright. All rights reserved. 

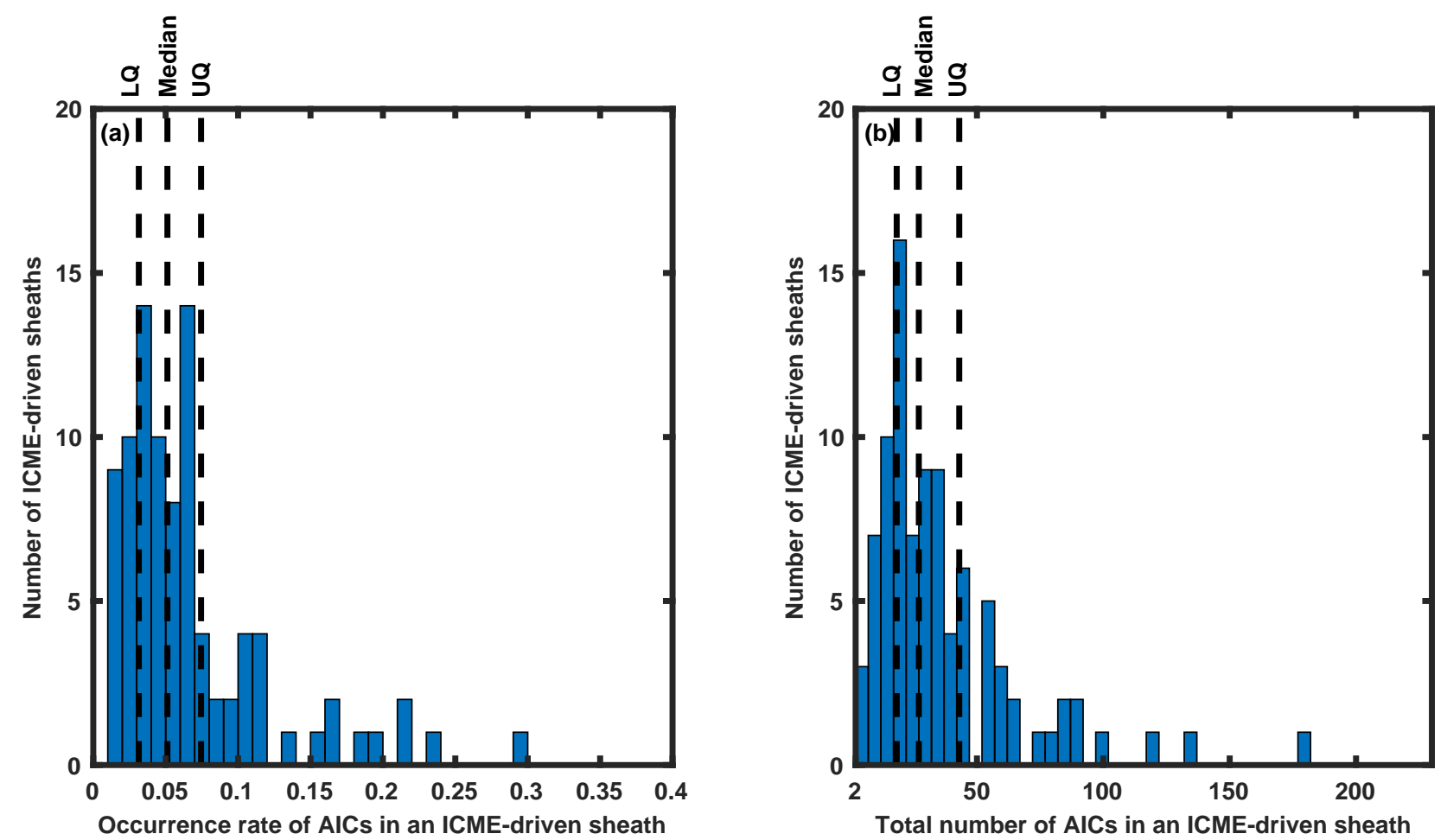

This article is protected by copyright. All rights reserved. 
Figure 5.
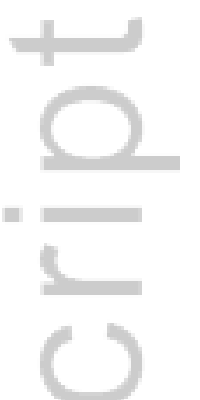

$\infty$
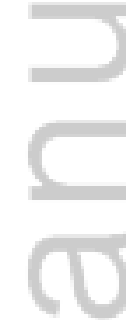

This article is protected by copyright. All rights reserved. 


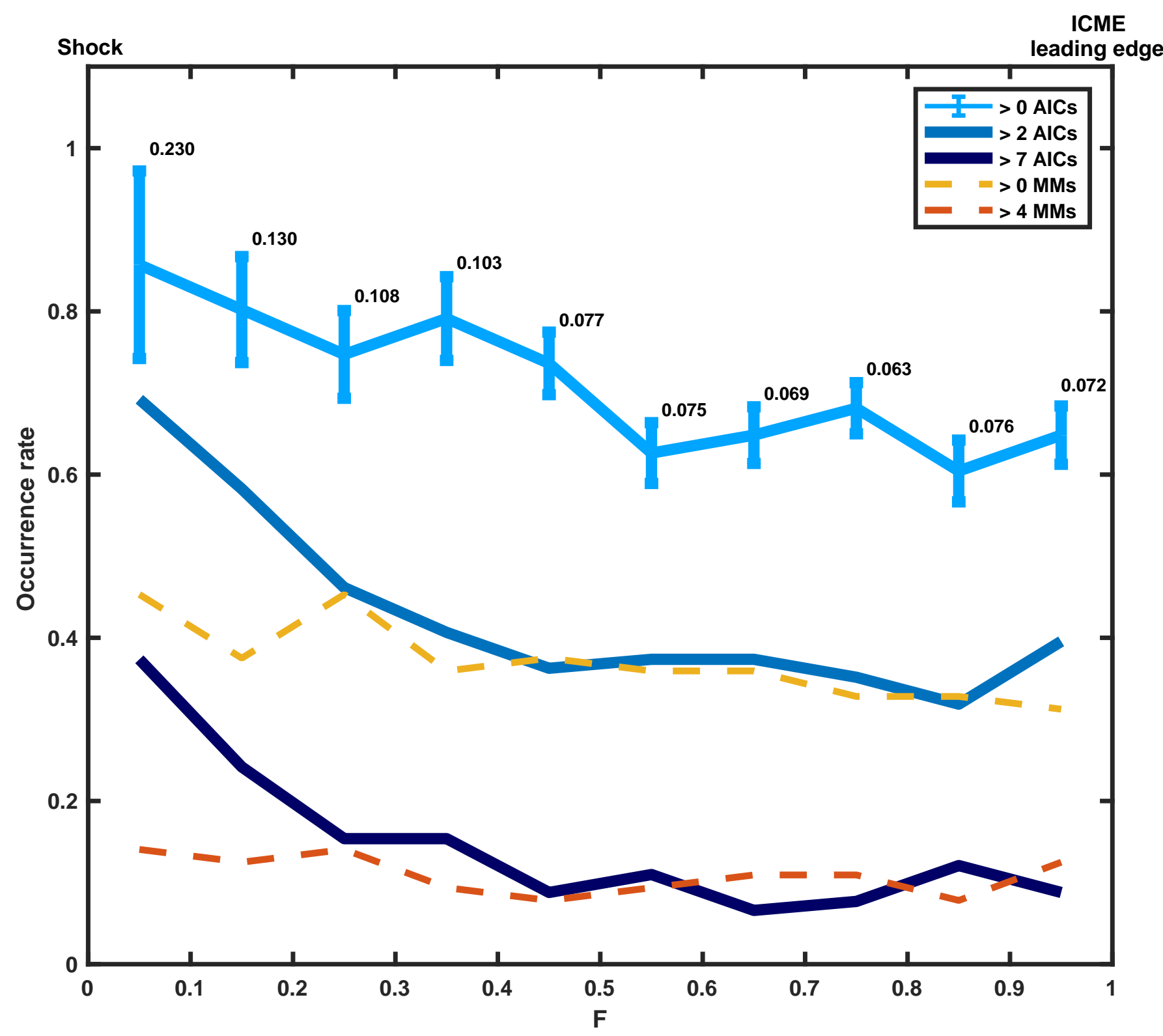

This article is protected by copyright. All rights reserved. 

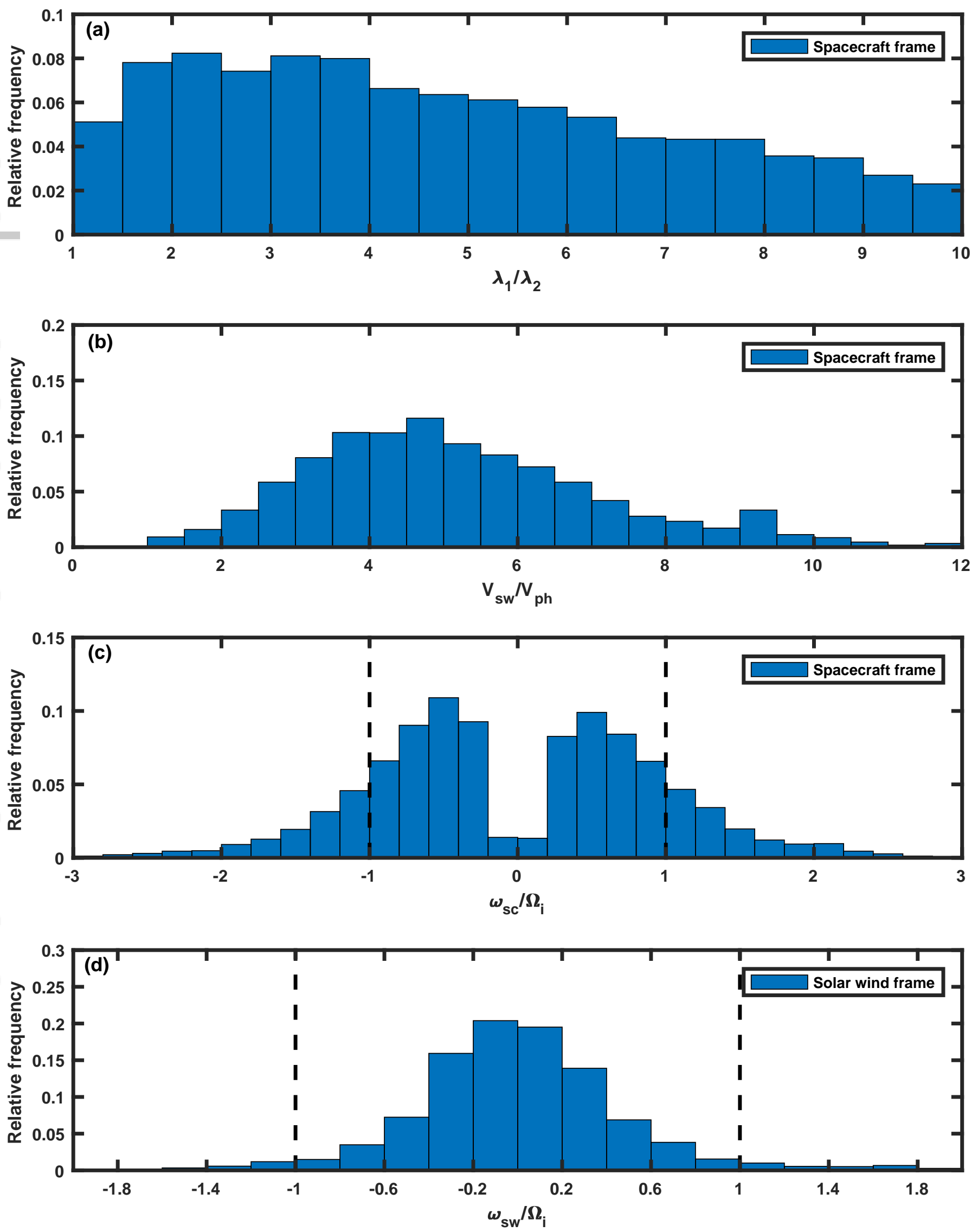

This article is protected by copyright. All rights reserved. 

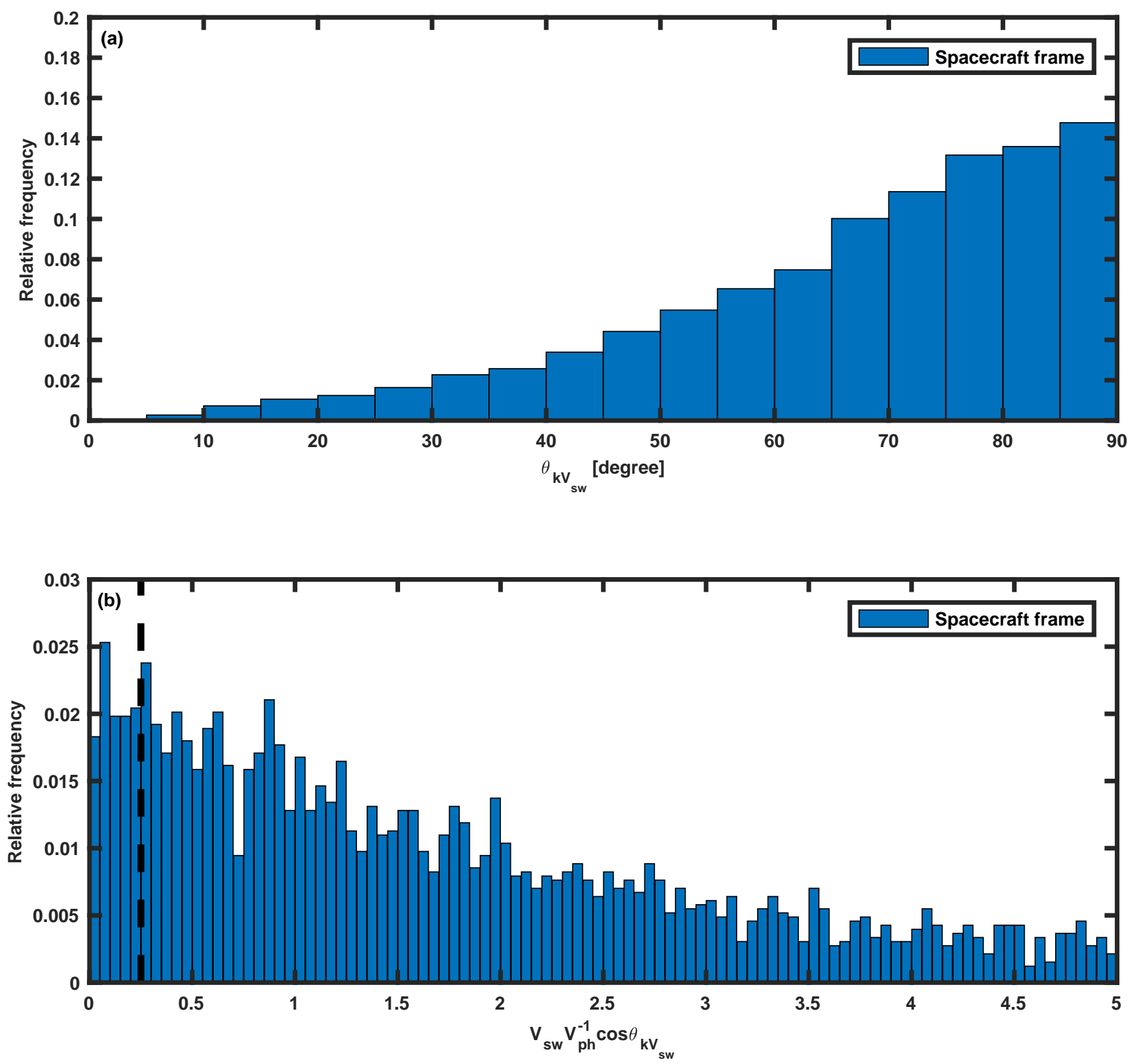

This article is protected by copyright. All rights reserved. 


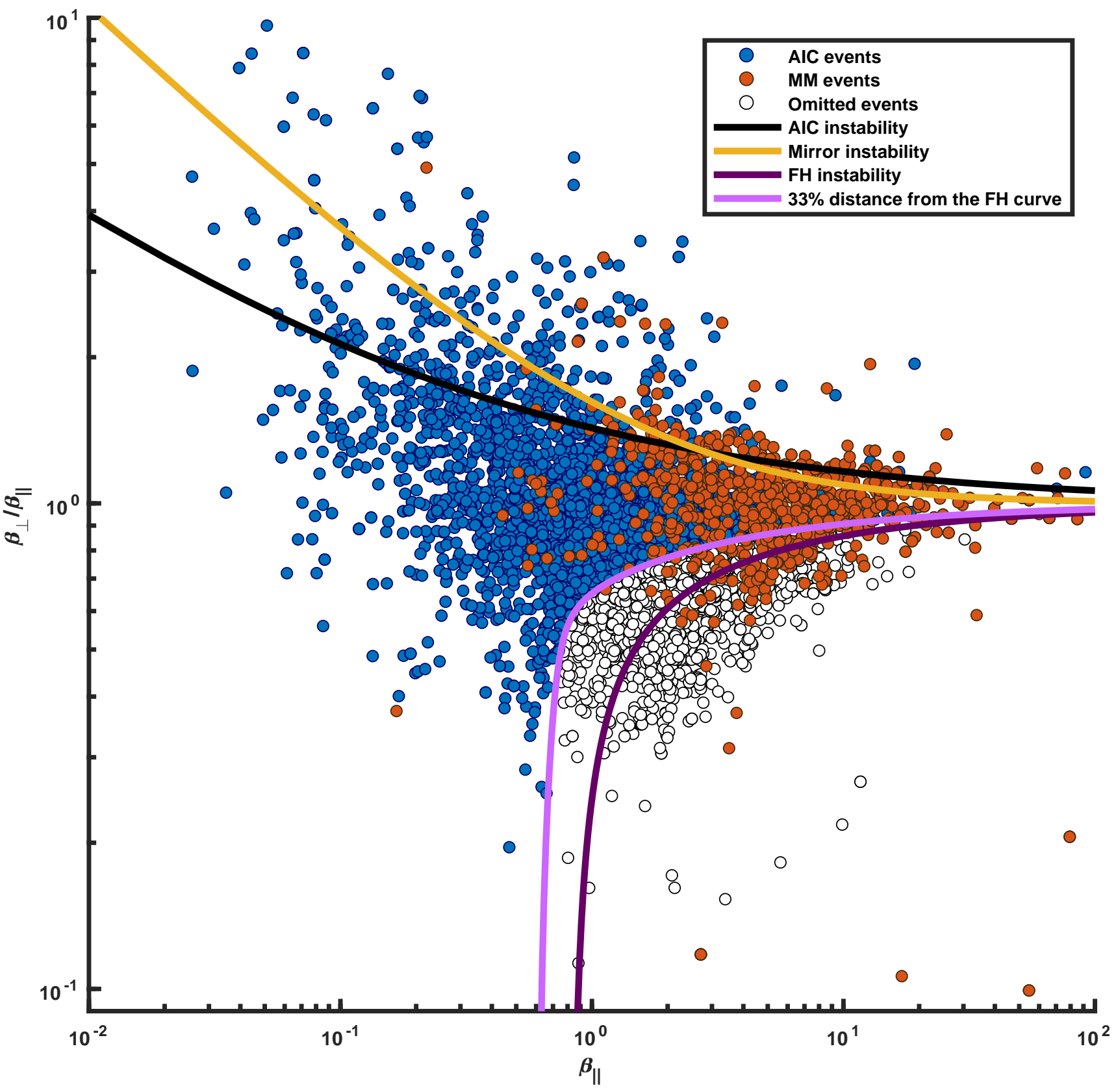

This article is protected by copyright. All rights reserved. 
Figure 9.
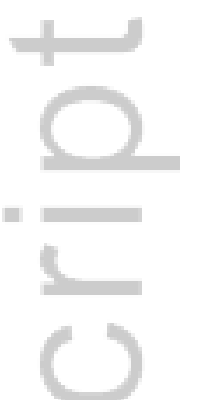

$\infty$
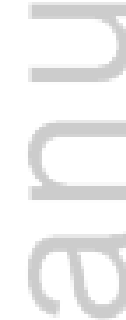

This article is protected by copyright. All rights reserved. 


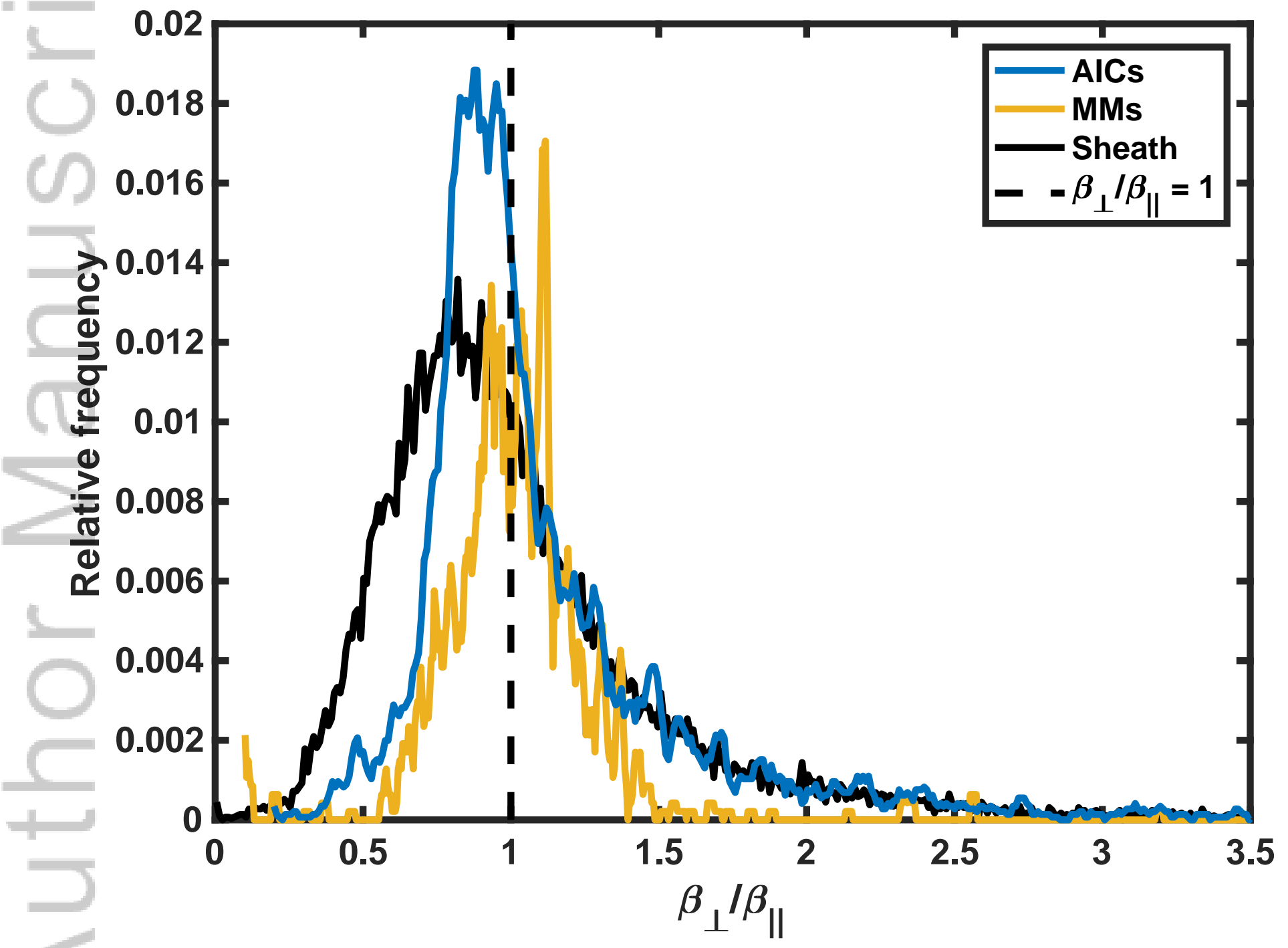

This article is protected by copyright. All rights reserved. 

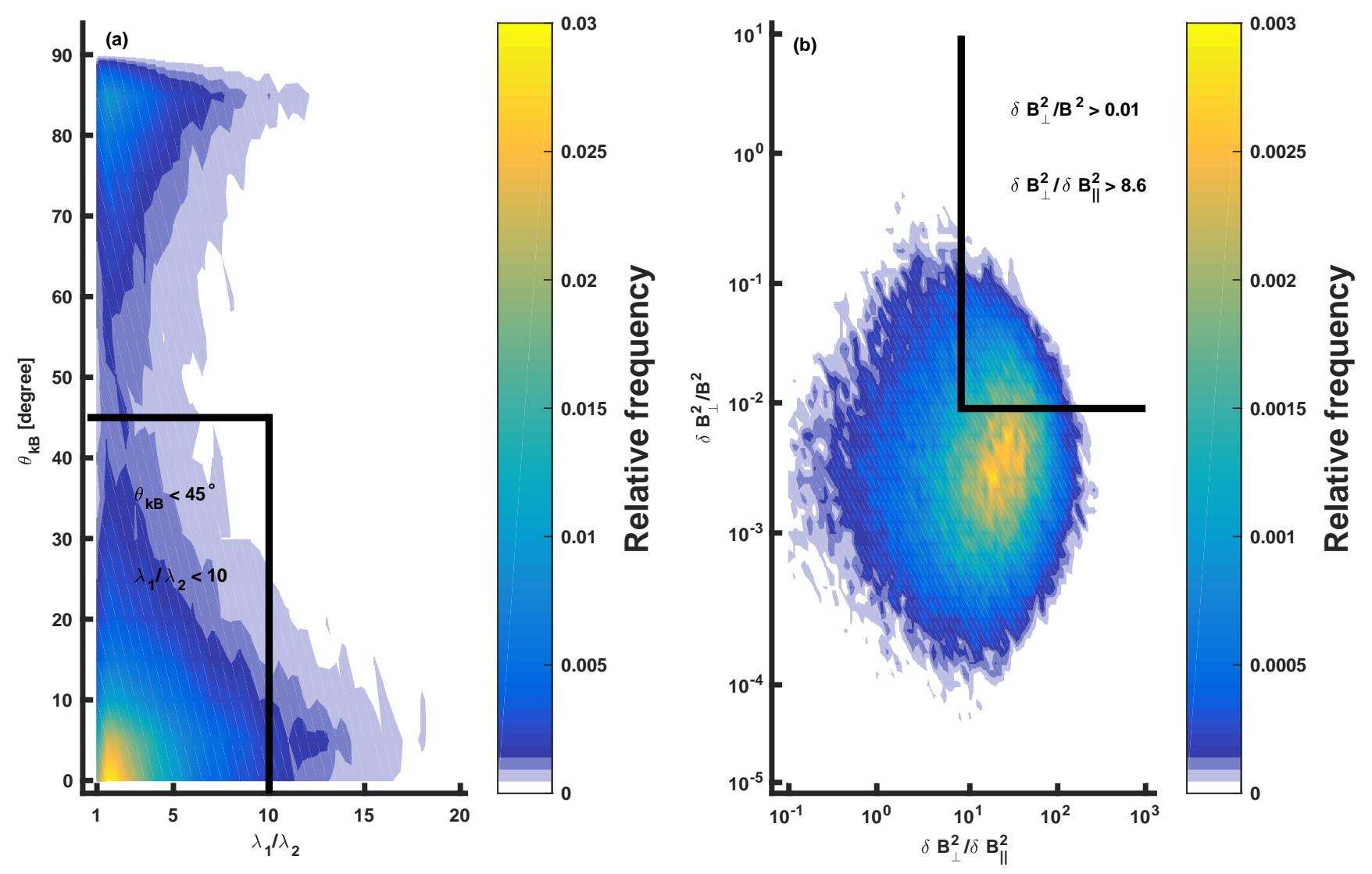


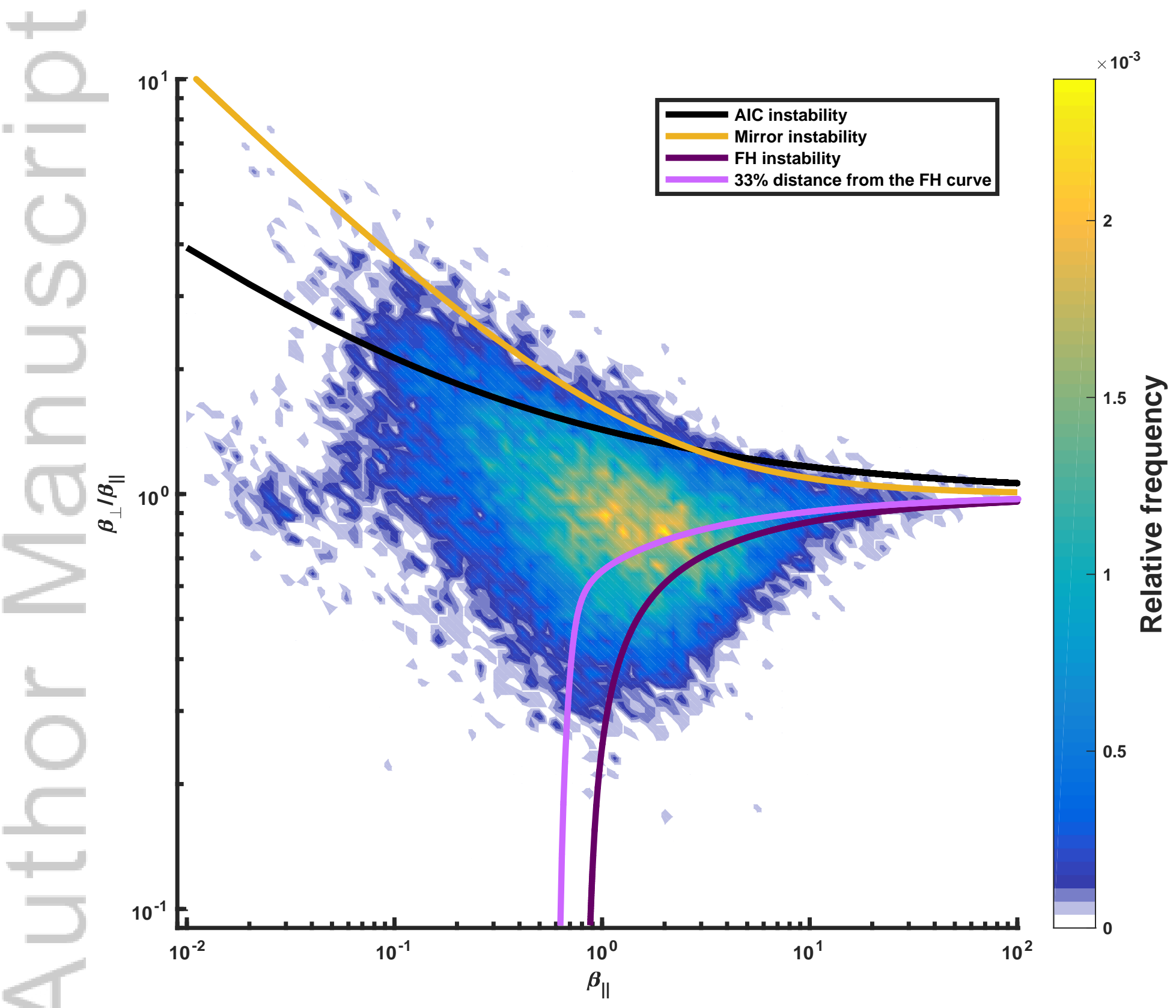

This article is protected by copyright. All rights reserved. 

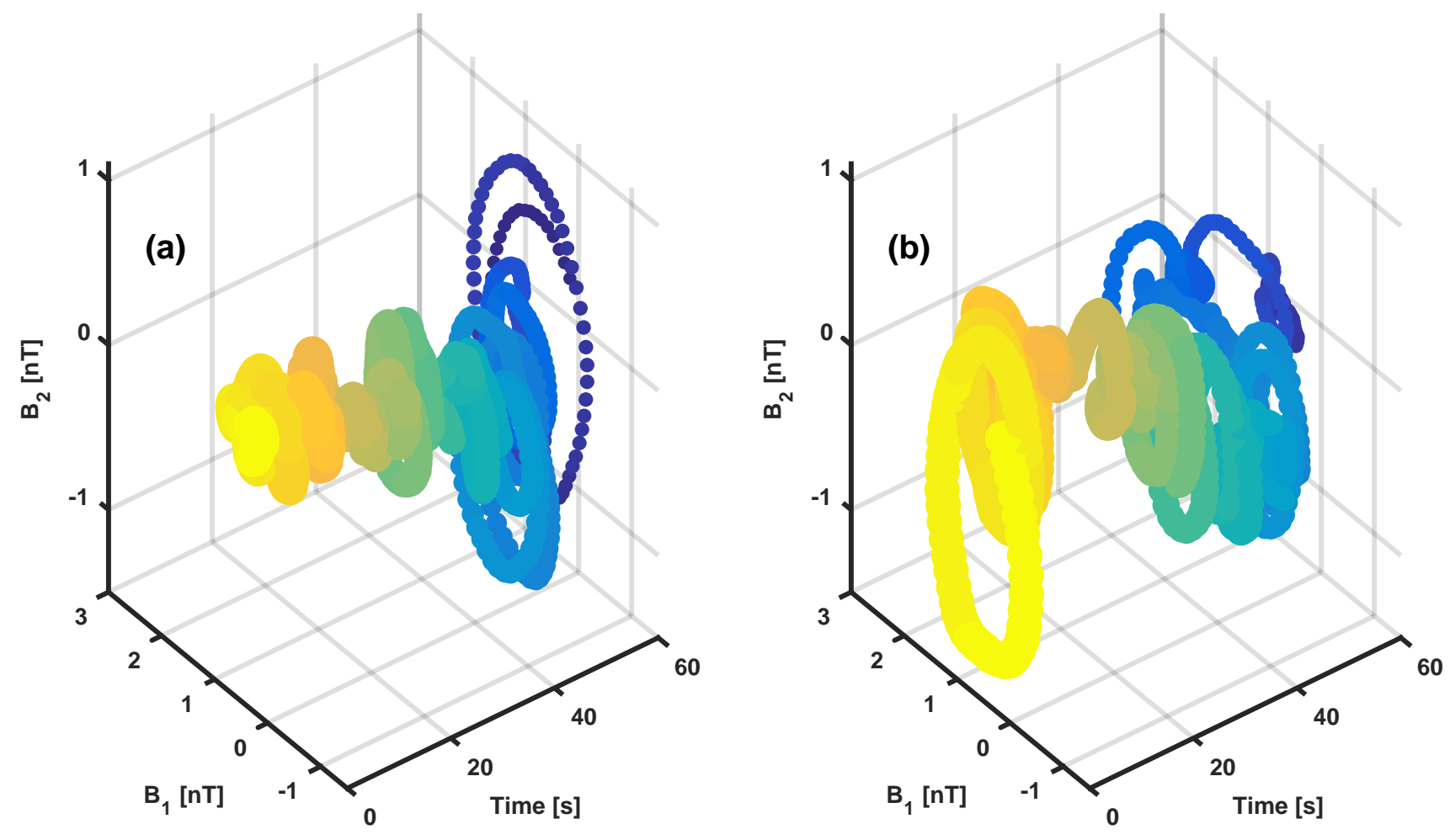

This article is protected by copyright. All rights reserved. 

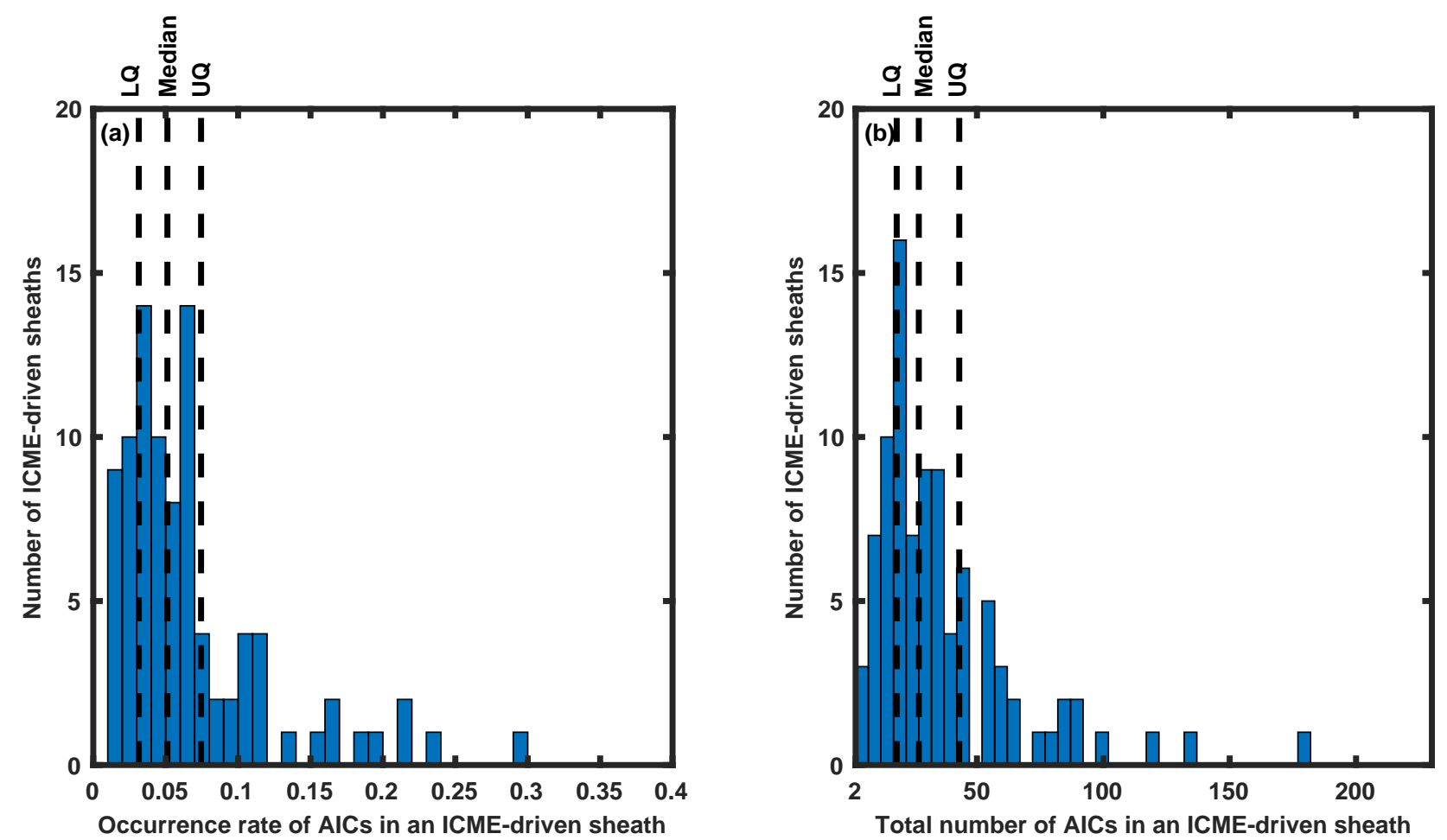

This article is protected by copyright. All rights reserved. 


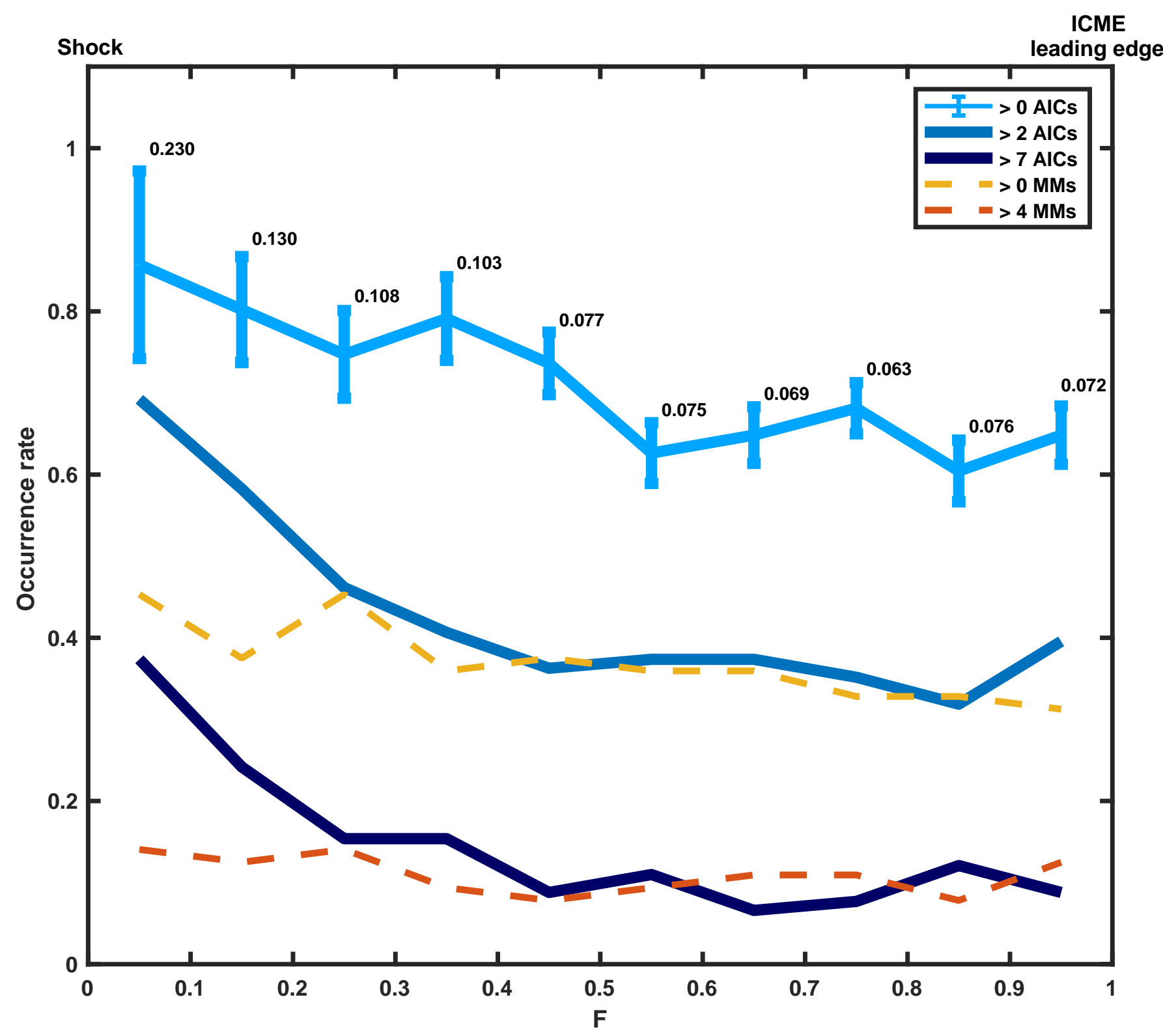

This article is protected by copyright. All rights reserved. 

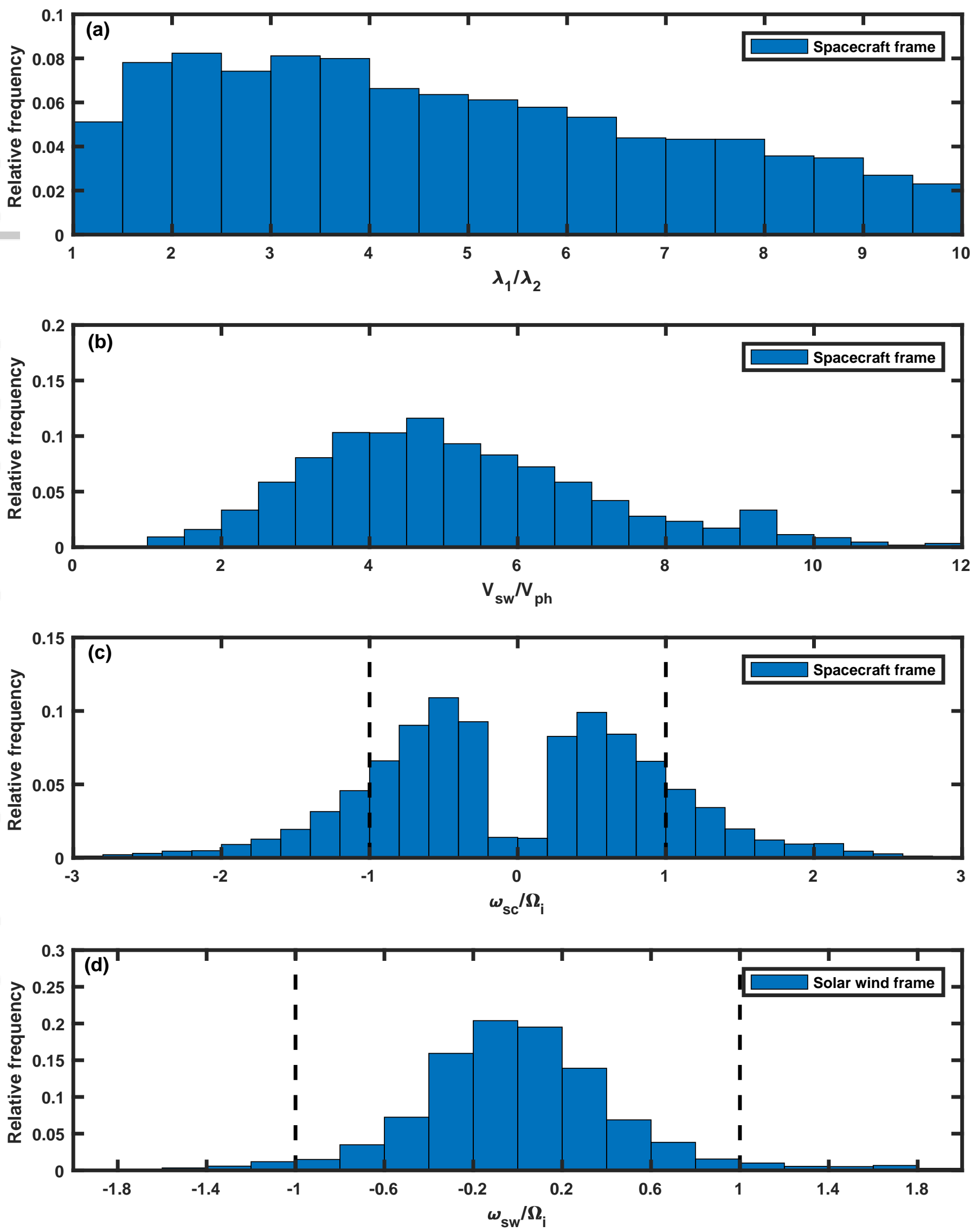

This article is protected by copyright. All rights reserved. 

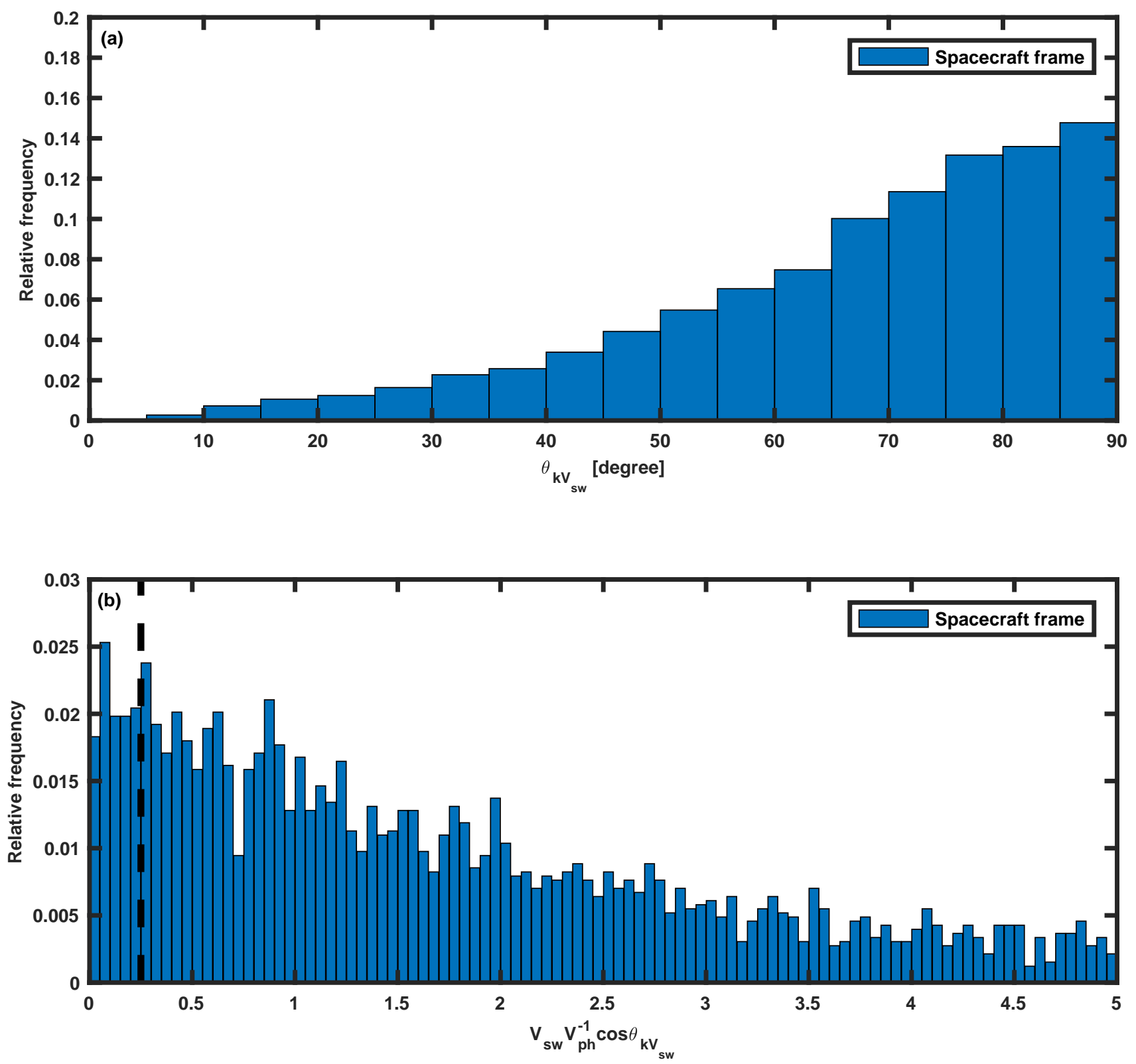

This article is protected by copyright. All rights reserved. 


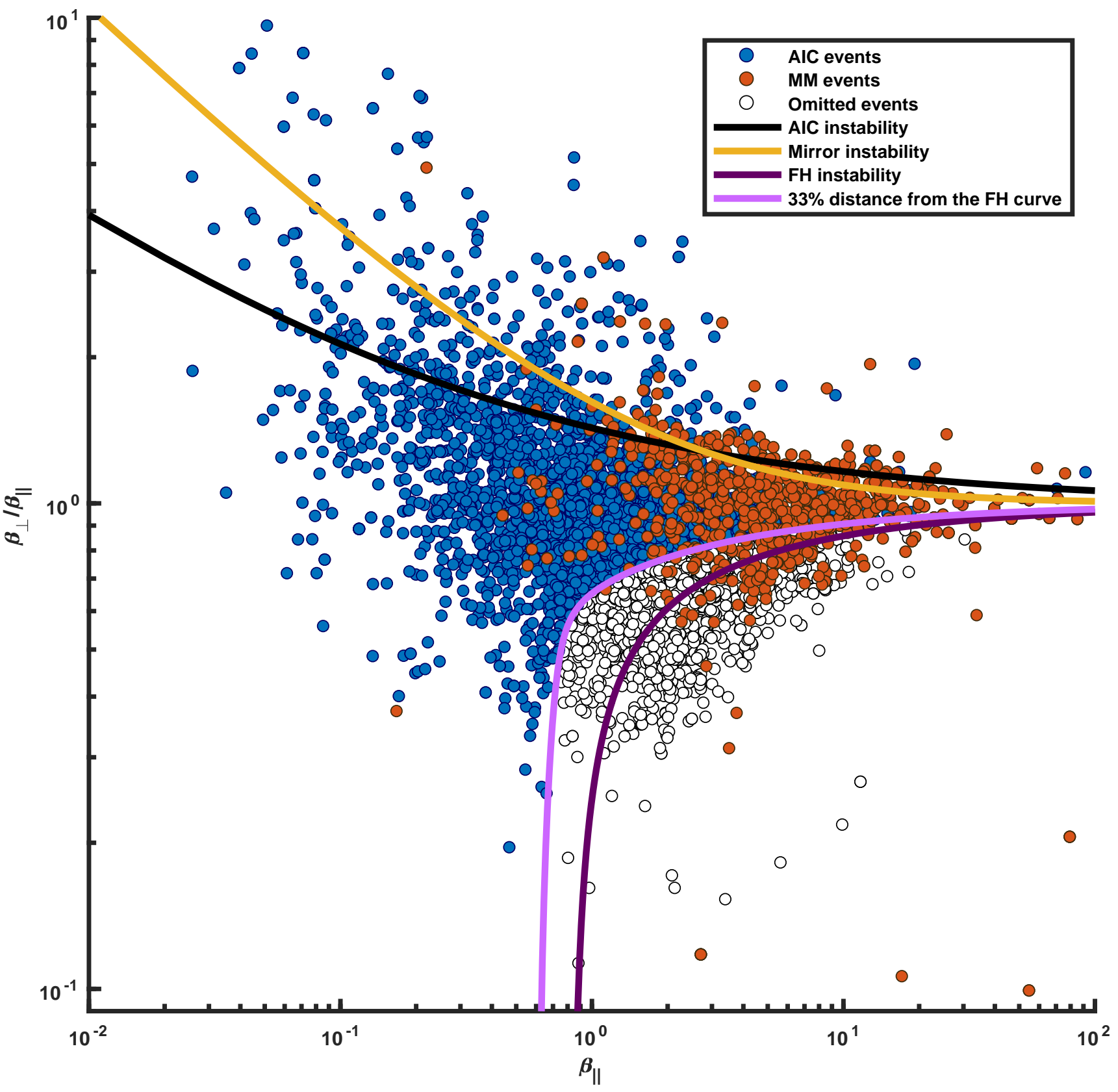

This article is protected by copyright. All rights reserved. 


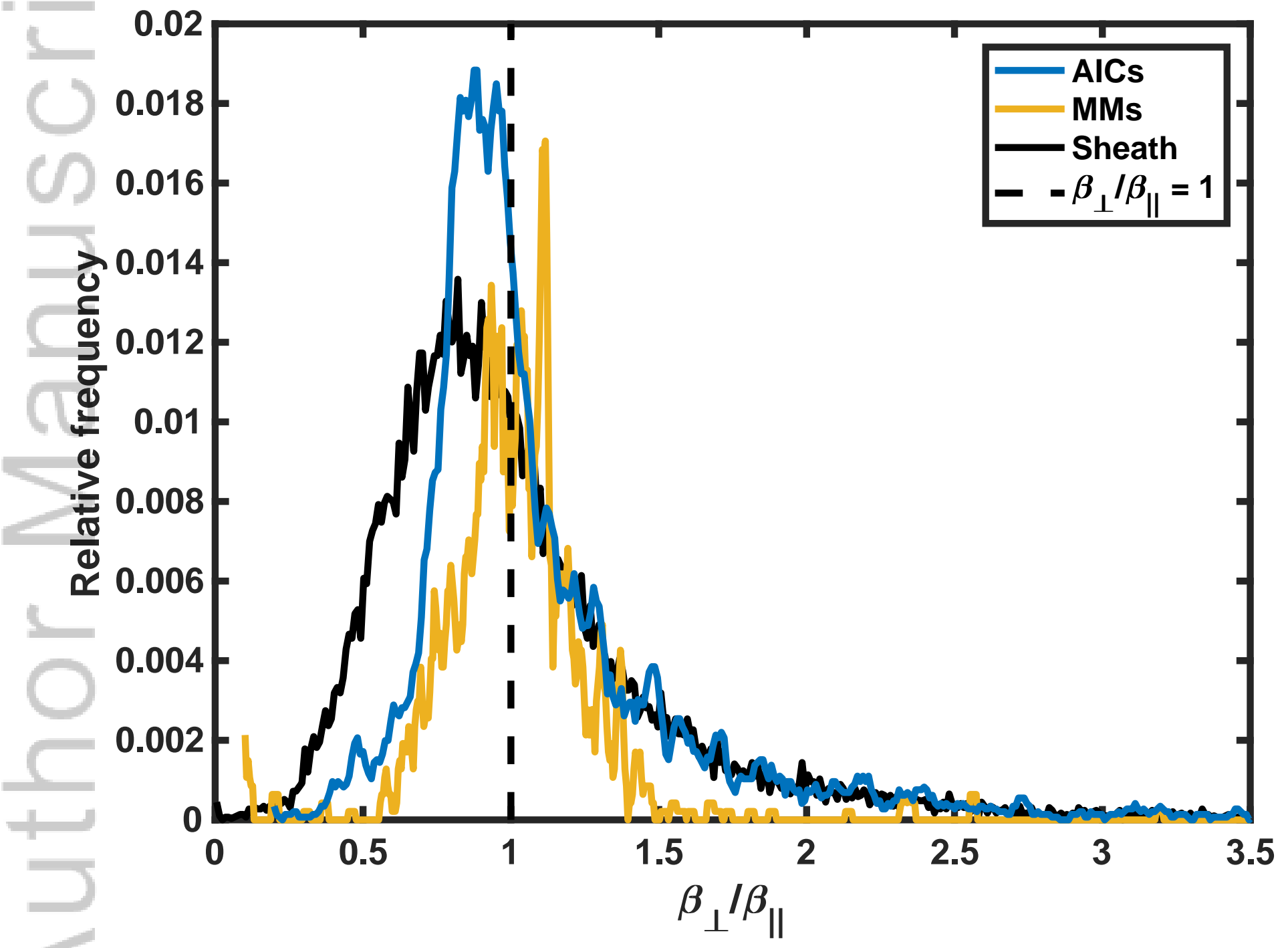

This article is protected by copyright. All rights reserved. 